\title{
Nuclear Equation of State in the Relativistic Point-Coupling Model Constrained by Excitations in Finite Nuclei
}

\author{
Esra Yüksel ${ }^{1}$ (D) Tomohiro Oishi ${ }^{2}$ and Nils Paar ${ }^{2, *(D)}$ \\ 1 Department of Physics, Faculty of Science and Letters, Davutpasa Campus, Yildiz Technical University, \\ Esenler, 34220 Istanbul, Turkey; eyuksel@yildiz.edu.tr \\ 2 Department of Physics, Faculty of Science, University of Zagreb, Bijenička c. 32, 10000 Zagreb, Croatia; \\ toishi@phy.hr \\ * Correspondence: npaar@phy.hr
}

Citation: Yüksel, E.; Oishi, T.; Paar, N. Nuclear Equation of State in the Relativistic Point-Coupling Model Constrained by Excitations in Finite Nuclei. Universe 2021, 7, 71. https:// doi.org/10.3390/universe7030071

Academic Editors: David Blaschke, Konstantin Maslov, Elena Litvinova and Evgeni Kolomeitsev

Received: 28 February 2021

Accepted: 17 March 2021

Published: 19 March 2021

Publisher's Note: MDPI stays neutral with regard to jurisdictional claims in published maps and institutional affiliations.

Copyright: (c) 2021 by the authors. Licensee MDPI, Basel, Switzerland. This article is an open access article distributed under the terms and conditions of the Creative Commons Attribution (CC BY) license (https:// creativecommons.org/licenses/by/ $4.0 /)$.

\begin{abstract}
Nuclear equation of state is often described in the framework of energy density functional. However, the isovector channel in most functionals has been poorly constrained, mainly due to rather limited available experimental data to probe it. Only recently, the relativistic nuclear energy density functional with an effective point-coupling interaction was constrained by supplementing the ground-state properties of nuclei with the experimental data on dipole polarizability and isoscalar monopole resonance energy in ${ }^{208} \mathrm{~Pb}$, resulting in DD-PCX parameterization. In this work, we pursue a complementary approach by introducing a family of 8 relativistic point-coupling functionals that reproduce the same nuclear ground-state properties, including binding energies and charge radii, but in addition have a constrained value of symmetry energy at saturation density in the range $J=29,30, \ldots, 36 \mathrm{MeV}$. In the next step, this family of functionals is employed in studies of excitation properties such as dipole polarizability and magnetic dipole transitions, and the respective experimental data are used to validate the optimal choice of functional as well as to assess reliable values of the symmetry energy and slope of the symmetry energy at saturation.
\end{abstract}

Keywords: nuclear energy density functional; equation of state; symmetry energy; nuclear excitations

\section{Introduction}

The construction of a universal energy density functional (EDF) that can make precise predictions on the properties of finite nuclei and can provide a reasonable description of nuclear matter properties is one of the major challenges in nuclear physics, and it is also relevant for nuclear astrophysics applications. The equation of state (EoS) of nuclear matter represents an essential ingredient for understanding many astrophysical phenomena, such as supernovae and neutron stars, as well as for the description of various nuclear processes in a stellar environment (for more details, see reviews [1,2]). In order to provide improved and universal nuclear energy density functionals as well as to improve their predictive power, many studies have been performed, e.g., see References [3-9]. The EoS around saturation density is often constrained by the ground-state properties of finite nuclei, and the nuclear excitation properties provide valuable information to optimize the EDFs used in the description of the EoS. More details about constraining the EoS from ground and collective excited states are given in an extensive review in Reference [10].

The general strategy in optimization of the functionals is to constrain their parameters in $\chi^{2}$ minimization using nuclear ground-state properties such as binding energies, charge radii, spin-orbit splittings, etc. The properties of symmetric nuclear matter around saturation are rather well-constrained by fitting the model parameters to the ground-state observables of nuclei. However, these observables are not able to constrain well the isovector channel of the functionals, which in turn leads to poorly constrained symmetry energy parameters of the EoS, and additional constraints on the pseudo-observables on the nuclear matter are introduced to cure this deficiency. Constraining the isovector channel of the 
EDFs with appropriate tools or observables is one of the principal aims of the nuclear physics community to obtain reliable information about the symmetry energy parameters around the saturation density as well as the properties of neutron-rich nuclei. In recent years, many works have been devoted to determining reliable values for the symmetry energy $J$, the slope of the symmetry energy $L$, and incompressibility of nuclear matter $K_{0}$ around the saturation density (see Reference [10] and the references therein) as well as finding possible observables to constrain their values [11-15]. These studies have shown that the symmetry energy parameter $J$ is expected between 31 and $32 \mathrm{MeV}$ while the slope of the symmetry energy $L$ is obtained between 30 and $90 \mathrm{MeV}$, and it is subject to larger uncertainties than the $J$ value. Moreover, the incompressibility parameter $K_{0}$, which is known as the curvature of the nuclear matter EoS, is predicted to be between 210 and $250 \mathrm{MeV}[10,15]$. There are also studies indicating larger incompressibilities if the ratio of the surface and volume coefficients $c=K_{\text {surf }} / K_{\text {vol }}$ from leptodermous expansion in powers of $A^{-1 / 3}$ of the incompressibility of finite nuclei $K_{A}$ is allowed to vary [16]. The decription of the EoS at higher densities represents a great challenge for ongoing research. Heavy-ion collisions can compress nuclear matter to densities present in neutron stars and core-collapse supernovae and, thus, can provide additional constraints of the EoS at higher densities [17].

Recent studies have shown that the isovector channel of the EDFs is strongly correlated with the neutron skin thickness, neutron star mass-radii, and isovector dipole excitation properties of nuclei and that these observables can be used to constrain the isovector channel of the functionals (see References [10,12,18] and the references therein). There are recent experimental constraints on the neutron skin thickness, obtained from the parity violating elastic electron scattering on ${ }^{208} \mathrm{~Pb}$ (PREX) [19]; however, the experimental uncertainties despite recent improvements remain rather large [20]. Among relevant observables, the isovector dipole excitations of nuclei have been known for a long time and represent a very useful constraint for optimization of the functionals. The dipole polarizability of nuclei, which is calculated from the isovector dipole excitation spectra, can also be used as a reliable observable to constrain the isovector channel and symmetry energy parameters of the nuclear EoS around the saturation density [21]. Charge-exchange transitions in nuclei also provide useful constraints for the isovector channel and the symmetry energy of the EDFs, in particular isobaric analog states [22], Gamow-Teller resonances [23], and anti-analog giant dipole resonances [24,25].

Recently, a new parameterization for the relativistic point-coupling interaction, denoted as DD-PCX [26], was optimized directly in $\chi^{2}$ minimization using the isovector dipole and isoscalar monopole excitation properties of ${ }^{208} \mathrm{~Pb}$ along with the nuclear groundstate properties of a set of nuclei. To this aim, a unified framework of the relativistic Hartree-Bogoliubov (RHB) model and self-consistent relativistic quasiparticle random phase approximation $(\mathrm{R}(\mathrm{Q}) \mathrm{RPA})$ was used in the optimization procedure and further analysis [26,27]. Using the collective excitation properties of ${ }^{208} \mathrm{~Pb}$, both the isoscalar and isovector channels of the EDF were constrained in a unique way, in which the symmetry energy and the slope of the symmetry energy around the saturation density were obtained as $J=31.12 \pm 0.32 \mathrm{MeV}$ and $L=46.32 \pm 1.68 \mathrm{MeV}$, respectively. These values are also consistent with the model predictions from various approaches given in References $[10,18]$. Including the constrained isoscalar giant monopole resonance (ISGMR) energy in the optimization of the DD-PCX functional, the incompressibility of nuclear matter was also obtained as $K_{0}=213.03 \pm 3.54 \mathrm{MeV}$, which is also consistent with the predictions of previous studies $[10,15]$.

The aim of this work is to introduce a complementary approach to constrain the symmetry energy parameters using relativistic EDFs with point-coupling interactions. First, a family of 8 functionals was established, which reproduced the same nuclear groundstate properties for a given set of nuclei, whereas the symmetry energy at the saturation density of the EoS was constrained to the values in the range of $J=29,30, \ldots, 36 \mathrm{MeV}$ in the $\chi^{2}$ minimization procedure. Furthermore, this family of functionals was used in studies of 
excitation properties such as dipole polarizability and magnetic dipole transitions, and by using respective experimental data, the optimal choice of the functional is discussed. In this way, insights into the values of the symmetry energy and slope of the symmetry energy at saturation are obtained. The results of the present study are also analyzed in comparison to the existing point-coupling functionals, DD-PC1 and DD-PCX, as well as with previous studies based on different EDFs [21,28]. Various families of the functionals have already been established both in the nonrelativistic and relativistic framework. [10,21,28]. Since the respective results are subject to considerable model dependence, it is important to provide several independent families of the EDFs. The isovector properties of the EDFs are also closely related to the size of the neutron skin thickness $\Delta R_{n p}$. Thus, the relation of the excitation properties with the $\Delta R_{n p}$ values is also explored in the present study.

This paper is organized in the following way. In Section 2, the basic formalism of the effective relativistic point-coupling interaction is introduced. Section 3 includes the results of the present analysis of the neutron skin thickness as well as the electric and magnetic dipole transitions and their relation to the symmetry energy properties. Finally, in Section 4, the conclusions of the present study are given.

\section{Relativistic Point-Coupling Functionals for Studies of the Symmetry Energy}

Among the variety of EDFs currently used in the description of static and dynamic properties of finite nuclei along the nuclide map as well as the nuclear EoS [10], the relativistic EDF represents one of successful frameworks [29]. It has already been employed in the description of various nuclear excitation properties [30-36] and astrophysically relevant weak interaction processes [27,37-41]. The theoretical framework used in this work is based on a self-consistent relativistic mean-field model with density dependent point-coupling interaction. It is derived from the effective Lagrangian density including the isoscalar-scalar, isoscalar-vector, and isovector-vector four-fermion interactions [4,29]:

$$
\begin{aligned}
\mathcal{L} & =\bar{\psi}(i \gamma \cdot \partial-m) \psi \\
& -\frac{1}{2} a_{S}(\hat{\rho})(\bar{\psi} \psi)(\bar{\psi} \psi)-\frac{1}{2} a_{V}(\hat{\rho})\left(\bar{\psi} \gamma^{\mu} \psi\right)\left(\bar{\psi} \gamma_{\mu} \psi\right)-\frac{1}{2} a_{T V}(\hat{\rho})\left(\bar{\psi} \vec{\tau} \gamma^{\mu} \psi\right)\left(\bar{\psi} \vec{\tau} \gamma_{\mu} \psi\right) \\
& -\frac{1}{2} \delta_{S}\left(\partial_{\nu} \bar{\psi} \psi\right)\left(\partial^{v} \bar{\psi} \psi\right)-e \bar{\psi} \gamma \cdot A \frac{\left(1-\tau_{3}\right)}{2} \psi .
\end{aligned}
$$

In addition to the free-nucleon terms, the Lagrangian density includes point-coupling interaction terms and coupling of protons to the electromagnetic field. The derivative term accounting for the leading effects of finite-range interactions is also necessary for a quantitative description of nuclear density distribution and radii. The couplings of the interaction terms $a_{s}, a_{v}$, and $a_{T V}$ are functionals of the vector density $\rho_{v}=\sqrt{j_{\mu} j^{\mu}}$ with the nucleon four-current $j^{\mu}=\bar{\psi} \gamma_{\mu} \psi$ [29]. The Dirac equation for the nucleons is obtained from the variation of the Lagrangian density (1) with respect to $\bar{\psi}$ [29]:

$$
\left[\gamma_{\mu}\left(i \partial^{\mu}-\Sigma^{\mu}-\Sigma_{R}^{\mu}-\vec{\tau} \Sigma_{T V}^{\mu}\right)-\left(m+\Sigma_{S}\right)\right] \psi=0,
$$

with the nucleon self-energies defined by the following:

$$
\begin{aligned}
\Sigma^{\mu} & =a_{V}\left(\rho_{v}\right) j^{\mu}+e \frac{\left(1-\tau_{3}\right)}{2} A^{\mu} \\
\Sigma_{S} & =a_{S}\left(\rho_{v}\right) \rho_{S}-\delta_{S} \square \rho_{S} \\
\Sigma_{R}^{\mu} & =\frac{1}{2} \frac{j^{\mu}}{\rho_{v}}\left\{\frac{\partial a_{S}}{\partial \rho} \rho_{S}^{2}+\frac{\partial a_{V}}{\partial \rho} j_{\mu} j^{\mu}+\frac{\partial a_{T V}}{\partial \rho} \vec{j}_{\mu} \vec{j}^{\mu}\right\} \\
\Sigma_{T V}^{\mu} & =a_{T V}\left(\rho_{v}\right) \vec{j}^{\mu},
\end{aligned}
$$

where $\Sigma^{\mu}, \Sigma_{S}$, and $\Sigma_{T V}^{\mu}$ denote the isoscalar-vector, isoscalar-scalar, and isovector-vector self-energies, respectively. The isoscalar-vector self-energy $\Sigma^{\mu}$ also includes the "rear- 
rangement" terms $\Sigma_{R}^{\mu}$, which are essential for energy-momentum conservation and the thermodynamical consistency of the model [42]. The currents and densities are given in more details in Reference [29].

For the functional form of the couplings, the following practical ansatz is chosen [4,29]:

$$
a_{i}(\rho)=a_{i}+\left(b_{i}+c_{i} x\right) e^{-d_{i} x} \quad(i \equiv S, V, T V),
$$

with $x=\rho / \rho_{\text {sat }}$ and $\rho_{\text {sat }}$ denoting the nucleon density at saturation in symmetric nuclear matter. The point-coupling model has ten parameters: $a_{S}, b_{S}, c_{S}, d_{S}, a_{V}, b_{V}, d_{V}, b_{T V}, d_{T V}$, and $\delta_{S}$. Following Reference [29], the parameters $c_{V}, a_{T V}$, and $c_{T V}$ are set to zero. In order to describe the properties of open-shell nuclei, the relativistic Hartree-Bogoliubov (RHB) model [29] is used. The pairing field is formulated using separable pairing force, which also includes two parameters for the pairing strength $\left(G_{p}\right.$ and $\left.G_{n}\right)$ [43].

In addition to the nuclear ground-state and excitation properties, the relativistic EDF can also be employed in the description of nuclear matter properties. In the translationally invariant infinite nuclear matter, the derivative term in Equation (1) drop out and the electromagnetic field is neglected [44]. More details about the implementation of the density dependent point-coupling interaction in nuclear matter description are given in Reference [44].

The binding energy per particle around the saturation density can be expanded as power series around $\delta \rightarrow 0$ [10],

$$
\frac{E}{A}(\rho, \delta)=\frac{E}{A}(\rho, 0)+S(\rho) \delta^{2}+\mathcal{O}\left[\delta^{4}\right]
$$

where the isospin asymmetry term is $\delta=\left(\rho^{n}-\rho^{p}\right) /\left(\rho^{n}+\rho^{p}\right)$. The first term of Equation (8) is the EoS of symmetric nuclear matter, given by

$$
\frac{E(\rho, 0)}{A}=\frac{E\left(\rho_{0}, 0\right)}{A}+\frac{1}{2} K_{0}\left(\frac{\rho-\rho_{0}}{3 \rho_{0}}\right)^{2}+\mathcal{O}\left[\left(\rho-\rho_{0}\right)^{3}\right],
$$

where $K_{0}$ represents the incompressibility of symmetric nuclear matter and is given by $\left.K_{0} \equiv 9 \rho_{0}^{2} \frac{\partial^{2} \frac{E}{A}(\rho, 0)}{\partial \rho^{2}}\right|_{\rho=\rho_{0}}$. The second term of Equation (8) corresponds to the symmetry energy and can be expanded in terms of the density,

$$
S(\rho)=J+L\left(\frac{\rho-\rho_{0}}{3 \rho_{0}}\right)+\frac{1}{2} K_{s y m}\left(\frac{\rho-\rho_{0}}{3 \rho_{0}}\right)^{2}+\mathcal{O}\left[\left(\rho-\rho_{0}\right)^{3}\right],
$$

where $J \equiv S\left(\rho_{0}\right)$ corresponds to the symmetry energy at saturation density, $\left.L \equiv 3 \rho_{0} \frac{S(\rho)}{\partial \rho}\right|_{\rho=\rho_{0}}$ is the slope of the symmetry energy at saturation, and $\left.K_{s y m} \equiv 9 \rho_{0}^{2} \frac{S(\rho)}{\partial \rho^{2}}\right|_{\rho=\rho_{0}}$ is the incompressibility of the symmetry energy at saturation. The symmetry energy properties will be discussed in the following section in relation to electric and magnetic excitations in finite nuclei.

In Reference [26] the relativistic point-coupling interaction DD-PCX has been constrained using the properties of isoscalar giant monopole resonance (ISGMR) energy and dipole polarizability of ${ }^{208} \mathrm{~Pb}$ along with the nuclear ground-state properties: binding energies (34 nuclei), charge radii (26 nuclei), and mean pairing gaps (15 nuclei) of the selected open-shell nuclei. In addition to the ground-state properties of nuclei, the excitation data have been used as an additional constraint for the relevant properties of the nuclear matter, incompressibility parameter $\left(K_{0}\right)$, symmetry energy $(J)$, and slope of the symmetry energy $(L)$ at saturation. In this work, a complementary approach is used, i.e., a set of 8 density-dependent point-coupling functionals is established by $\chi^{2}$ minimization, using not only the given ground-state properties of nuclei but also an additional constraint on the symmetry energy at the saturation in the range of values, $J=29-36 \mathrm{MeV}$. In optimization of 
the interaction, the average neutron and proton pairing gap values are calculated using [45]

$$
\langle u v \Delta\rangle_{p, n}=\frac{\sum_{k} u_{k} v_{k} \Delta_{k}}{\sum_{k} u_{k} v_{k}}
$$

where $v$ and $u$ are occupation factors of the single-particle states and $\Delta_{k}$ is the state dependent single-particle gap. On the other side, the pairing gaps were extracted from the experimental data on binding energies using a five-point formula [28].

In constraining the model parameters of the DD-PC family of functionals, the following experimental data on nuclear ground-state properties from light to heavy nuclei were used: binding energies (72 nuclei), charge radii (36 nuclei), diffraction radii (22 nuclei), surface thickness (18 nuclei), neutron pairing gaps (15 nuclei), and proton pairing gaps (14 nuclei) [28]. In Table 1, the selected nuclei are presented alongside the relevant data used in the fitting of the functionals. A similar choice of observables and the set of experimental data were adopted in adjusting the model parameters of Skyrme functionals in Reference [28]. In the present calculation, the adopted errors for the binding energies, charge radii, diffraction radii, surface thickness, and pairing gaps were taken as $1.0 \mathrm{MeV}$, $0.02 \mathrm{fm}, 0.05 \mathrm{fm}, 0.05 \mathrm{fm}$, and $0.15 \mathrm{MeV}$, respectively. In addition, nuclear matter properties were constrained with the given adopted errors: saturation density $\rho_{\text {sat }}=0.152 \mathrm{fm}^{-3}(10 \%)$, binding energy at saturation $E / A=-16.14 \mathrm{MeV}(10 \%)$, effective mass $m^{*} / m=0.58(10 \%)$, and incompressibility parameter $K_{0}=230 \mathrm{MeV}(1 \%)$, as used in Reference [4]. For each member of the DD-PC interaction family, an additional constraint was imposed on the values of $J=29,30, \ldots, 36 \mathrm{MeV}$. The value of the proton (neutron) mass was taken as 939.565379 (938.272046) $\mathrm{MeV} / \mathrm{c}^{2}$.

Table 1. The experimental data for the binding energies (B.E.) (72 nuclei) [46,47] and charge radii $r_{c}$ (36 nuclei) [48] are used alongside with the neutron (15 nuclei) and proton (14 nuclei) mean gap values $\Delta_{n(p)}$, diffraction radius $r_{\text {diffr }}$ (22 nuclei), and surface thickness $\sigma$ (18 nuclei) values for the selected nuclei [28] in the fitting of the DD-PC $(J=29,30, \ldots, 36 \mathrm{MeV})$ functionals. In the second line, we display the adopted errors for each observable. The adopted error is also multiplied by a further integer weight factor that is given in the parentheses next to each observable.

\begin{tabular}{|c|c|c|c|c|c|c|}
\hline Nucleus & $\begin{array}{c}\Delta_{n} \\
( \pm 0.15 \mathrm{MeV})\end{array}$ & $\begin{array}{c}\Delta_{p} \\
( \pm 0.15 \mathrm{MeV})\end{array}$ & $\begin{array}{c}\text { B.E. } \\
( \pm 1 \mathrm{MeV})\end{array}$ & $\begin{array}{c}r_{c} \\
( \pm 0.02 \mathrm{fm})\end{array}$ & $\begin{array}{c}r_{d i f f r} \\
( \pm 0.05 \mathrm{fm})\end{array}$ & $\begin{array}{c}\sigma \\
( \pm 0.05 \mathrm{fm})\end{array}$ \\
\hline${ }^{16} \mathrm{O}$ & & & $-127.619(2)$ & & $2.777(1)$ & \\
\hline${ }^{18} \mathrm{O}$ & & & $-139.807(1)$ & & & \\
\hline${ }^{20} \mathrm{O}$ & & & $-151.371(1)$ & & & \\
\hline${ }^{22} \mathrm{O}$ & & & $-162.026(1)$ & & & \\
\hline${ }^{18} \mathrm{Ne}$ & & & $-132.142(1)$ & & & \\
\hline${ }^{20} \mathrm{Mg}$ & & & $-134.479(1)$ & & & \\
\hline${ }^{34} \mathrm{Si}^{\circ}$ & & & $-283.428(1)$ & & & \\
\hline${ }^{36} \mathrm{~S}$ & & $1.52(1)$ & $-308.714(1)$ & $3.299(1)$ & $3.577(1)$ & $0.994(2)$ \\
\hline${ }^{38} \mathrm{Ar}$ & & $1.44(1)$ & $-327.342(1)$ & $3.404(1)$ & & \\
\hline${ }^{36} \mathrm{Ca}$ & & & $-281.371(1)$ & & & \\
\hline${ }^{38} \mathrm{Ca}$ & & & $-313.121(1)$ & & & \\
\hline${ }^{40} \mathrm{Ca}$ & & & $-342.052(2)$ & $3.485(2)$ & $3.831(2)$ & $0.978(2)$ \\
\hline${ }^{42} \mathrm{Ca}$ & $1.68(1)$ & & $-361.895(1)$ & $3.513(2)$ & $3.876(2)$ & $0.999(2)$ \\
\hline${ }^{44} \mathrm{Ca}$ & $1.70(1)$ & & $-380.959(1)$ & $3.523(3)$ & $3.912(1)$ & $0.975(2)$ \\
\hline${ }^{46} \mathrm{Ca}$ & $1.49(1)$ & & $-398.772(1)$ & $3.502(1)$ & & \\
\hline${ }^{48} \mathrm{Ca}$ & & & $-416.001(1)$ & $3.484(1)$ & $3.936(1)$ & $0.881(1)$ \\
\hline${ }^{50} \mathrm{Ca}$ & & & $-427.508(1)$ & & & \\
\hline${ }^{42} \mathrm{Ti}$ & & & $-346.888(1)$ & & & \\
\hline${ }^{50} \mathrm{Ti}$ & & & $-437.784(1)$ & & & \\
\hline${ }^{52} \mathrm{Cr}$ & & & $-456.350(1)$ & $3.642(1)$ & $4.173(1)$ & $0.924(1)$ \\
\hline${ }^{54} \mathrm{Fe}$ & & & $-471.763(1)$ & $3.693(5)$ & $4.258(5)$ & $0.900(5)$ \\
\hline${ }^{56} \mathrm{Ni}$ & & & $-483.994(1)$ & & & \\
\hline${ }^{68} \mathrm{Ni}$ & & & $-590.407(1)$ & & & \\
\hline${ }^{72} \mathrm{Ni}$ & & & $-613.455(1)$ & & & \\
\hline${ }^{84} \mathrm{Se}$ & & & $-727.338(1)$ & & & \\
\hline${ }^{86} \mathrm{Kr}$ & & & $-749.234(1)$ & & & \\
\hline
\end{tabular}


Table 1. Cont.

\begin{tabular}{|c|c|c|c|c|c|c|}
\hline Nucleus & $\begin{array}{c}\Delta_{n} \\
( \pm 0.15 \mathrm{MeV})\end{array}$ & $\begin{array}{c}\Delta_{p} \\
( \pm 0.15 \mathrm{MeV})\end{array}$ & $\begin{array}{c}\text { B.E. } \\
( \pm 1 \mathrm{MeV})\end{array}$ & $\begin{array}{c}r_{c} \\
( \pm 0.02 \mathrm{fm})\end{array}$ & $\begin{array}{c}r_{d i f f r} \\
( \pm 0.05 \mathrm{fm})\end{array}$ & $\begin{array}{c}\sigma \\
( \pm 0.05 \mathrm{fm})\end{array}$ \\
\hline${ }^{88} \mathrm{Sr}$ & & & $-768.468(1)$ & $4.220(1)$ & $4.994(1)$ & $0.923(1)$ \\
\hline${ }^{90} \mathrm{Zr}$ & & & $-783.898(1)$ & $4.272(1)$ & $5.040(1)$ & $0.957(1)$ \\
\hline${ }^{92} \mathrm{Mo}$ & & $1.40(1)$ & $-796.510(1)$ & $4.315(1)$ & $5.104(1)$ & $0.950(1)$ \\
\hline${ }^{94} \mathrm{Ru}$ & & & $-806.864(1)$ & & & \\
\hline${ }^{96} \mathrm{Pd}$ & & & $-815.040(1)$ & & & \\
\hline${ }^{98} \mathrm{Cd}$ & & & $-821.072(1)$ & & & \\
\hline${ }^{100} \mathrm{Sn}$ & & & $-825.297(2)$ & & & \\
\hline${ }^{106} \mathrm{Sn}$ & & & $-893.795(1)$ & & & \\
\hline${ }^{108} \mathrm{Sn}$ & & & $-914.654(1)$ & & & \\
\hline${ }^{112} \mathrm{Sn}$ & $1.41(1)$ & & $-953.526(1)$ & $4.596(1)$ & $5.477(2)$ & \\
\hline${ }^{114} \mathrm{Sn}$ & $1.26(3)$ & & $-971.570(1)$ & $4.610(1)$ & $5.509(2)$ & $0.948(2)$ \\
\hline${ }^{116} \mathrm{Sn}$ & $1.21(3)$ & & -988.681 (1) & $4.626(1)$ & $5.541(1)$ & $0.945(1)$ \\
\hline${ }^{118} \mathrm{Sn}$ & $1.34(1)$ & & $-1004.951(1)$ & $4.640(1)$ & $5.571(1)$ & $0.931(1)$ \\
\hline${ }^{120} \mathrm{Sn}$ & $1.39(1)$ & & $-1020.539(1)$ & & $5.591(1)$ & \\
\hline${ }^{122} \mathrm{Sn}$ & $1.37(1)$ & & $-1035.524(1)$ & & & \\
\hline${ }^{124} \mathrm{Sn}$ & $1.31(1)$ & & $-1049.960(1)$ & $4.674(1)$ & $5.640(1)$ & \\
\hline${ }^{126} \mathrm{Sn}$ & $1.26(1)$ & & $-1063.883(1)$ & & & \\
\hline${ }^{128} \mathrm{Sn}$ & $1.22(1)$ & & $-1077.373(1)$ & & & \\
\hline${ }^{130} \mathrm{Sn}$ & $1.17(3)$ & & $-1090.286(1)$ & & & \\
\hline${ }^{132} \mathrm{Sn}$ & & & $-1102.840(1)$ & & & \\
\hline${ }^{134} \mathrm{Sn}$ & & & $-1108.871(1)$ & & & \\
\hline${ }^{134} \mathrm{Te}$ & & $0.81(1)$ & $-1123.410(1)$ & & & \\
\hline${ }^{136} \mathrm{Xe}$ & & $0.98(1)$ & $-1141.881(1)$ & & & \\
\hline${ }^{138} \mathrm{Ba}$ & & $1.12(1)$ & $-1158.292(1)$ & $4.834(1)$ & $5.868(2)$ & $0.900(1)$ \\
\hline${ }^{140} \mathrm{Ce}$ & & $1.21(1)$ & $-1172.687(1)$ & $4.877(1)$ & & \\
\hline${ }^{142} \mathrm{Nd}$ & & $1.23(1)$ & $-1185.136(1)$ & 4.915 (1) & $5.876(3)$ & $0.989(3)$ \\
\hline${ }^{144} \mathrm{Sm}$ & & $1.25(1)$ & $-1195.729(1)$ & & & \\
\hline${ }^{146} \mathrm{Gd}$ & & $1.42(1)$ & $-1204.427(1)$ & $4.984(1)$ & & \\
\hline${ }^{148} \mathrm{Dy}$ & & 1.49 (1) & $-1210.773(1)$ & $5.046(1)$ & & \\
\hline${ }^{150} \mathrm{Er}$ & & & $-1215.329(1)$ & $5.076(2)$ & & \\
\hline${ }^{152} \mathrm{Yb}$ & & & $-1218.396(1)$ & & & \\
\hline${ }^{206} \mathrm{Hg}$ & & & $-1621.048(1)$ & $5.485(1)$ & & \\
\hline${ }^{198} \mathrm{~Pb}$ & & & $-1560.018(1)$ & $5.450(2)$ & & \\
\hline${ }^{200} \mathrm{~Pb}$ & & & $-1576.361(1)$ & $5.459(2)$ & & \\
\hline${ }^{202} \mathrm{~Pb}$ & & & $-1592.193(1)$ & $5.474(1)$ & & \\
\hline${ }^{204} \mathrm{~Pb}$ & & & $-1607.505(1)$ & $5.483(1)$ & $6.749(1)$ & $0.918(1)$ \\
\hline${ }^{206} \mathrm{~Pb}$ & $0.59(1)$ & & $-1622.323(1)$ & $5.494(1)$ & $6.766(1)$ & $0.921(1)$ \\
\hline${ }^{208} \mathrm{~Pb}$ & & & $-1636.429(1)$ & $5.505(1)$ & $6.806(1)$ & $0.900(1)$ \\
\hline${ }^{210} \mathrm{~Pb}$ & $0.66(1)$ & & $-1645.552(1)$ & $5.523(1)$ & & \\
\hline${ }^{212} \mathrm{~Pb}$ & & & $-1654.514(1)$ & $5.542(1)$ & & \\
\hline${ }^{214} \mathrm{~Pb}$ & & & $-1663.290(1)$ & $5.562(1)$ & & \\
\hline${ }^{210} \mathrm{Po}$ & & $0.81(1)$ & $-1645.212(1)$ & $5.534(1)$ & & \\
\hline${ }^{212} \mathrm{Rn}$ & & $0.88(1)$ & $-1652.496(1)$ & $5.555(2)$ & & \\
\hline${ }^{214} \mathrm{Ra}$ & & $0.96(1)$ & $-1658.322(1)$ & $5.571(3)$ & & \\
\hline${ }^{216} \mathrm{Th}$ & & & $-1662.694(1)$ & & & \\
\hline${ }^{218} \mathrm{U}$ & & & $-1665.659(1)$ & & & \\
\hline
\end{tabular}

Although the same ground-state data were used systematically in the fitting protocol, the DD-PC functionals can accommodate a broad range of $J$ values for the symmetry energy while providing similar quality in the description of binding energies and radii. Table 2 shows the resulting parameter sets of 8 functionals from the DD-PC family with $J=29,30, \ldots, 36 \mathrm{MeV}$. In the next step, this set of functionals will be examined by using nuclear excitation data and the possibility to constrain the optimal range of the symmetry energy parameters will be investigated. 
Table 2. Parameters of the DD-PC interactions with different values of the symmetry energy at the saturation $J$, given in units of MeV.

\begin{tabular}{|c|c|c|c|c|c|c|c|c|}
\hline Parameters & $J=29$ & $J=30$ & $J=31$ & $J=32$ & $J=33$ & $J=34$ & $J=35$ & $J=36$ \\
\hline$a_{S}\left(\mathrm{fm}^{2}\right)$ & -10.418334630 & -10.384498639 & -10.390657606 & -10.389488800 & -10.387142700 & -10.386956740 & -10.384414509 & -10.387781497 \\
\hline$b_{S}\left(\mathrm{fm}^{2}\right)$ & -9.163612956 & -9.202629009 & -9.189174805 & -9.197715907 & -9.215314920 & -9.208074838 & -9.215072973 & -9.218990127 \\
\hline$c_{S}\left(\mathrm{fm}^{2}\right)$ & -4.968064171 & -5.014345965 & -5.031326770 & -5.030638408 & -5.021597419 & -5.023220520 & -5.012403311 & -5.019048957 \\
\hline$d_{S}$ & 1.348374834 & 1.351382727 & 1.352967739 & 1.352329476 & 1.351064617 & 1.352262229 & 1.350836047 & 1.352173708 \\
\hline$a_{V}\left(\mathrm{fm}^{2}\right)$ & 6.591051202 & 6.583857817 & 6.584374904 & 6.584425799 & 6.584907238 & 6.587404662 & 6.583350394 & 6.585576629 \\
\hline$b_{V}\left(\mathrm{fm}^{2}\right)$ & 8.366192199 & 8.380745134 & 8.363054275 & 8.358949669 & 8.366666526 & 8.350225226 & 8.361167422 & 8.359267286 \\
\hline$d_{V}$ & 0.737531721 & 0.742903470 & 0.740865993 & 0.739885318 & 0.740096759 & 0.740274812 & 0.740322161 & 0.740857140 \\
\hline$b_{T V}\left(\mathrm{fm}^{2}\right)$ & 4.370433886 & 3.630253620 & 3.017651110 & 2.581533741 & 2.200070930 & 1.913161650 & 1.654675062 & 1.474609051 \\
\hline$\delta_{S}\left(\mathrm{fm}^{4}\right)$ & -0.823980938 & -0.828843122 & -0.835583928 & -0.841884906 & -0.846057419 & -0.849721556 & -0.849999864 & -0.859856552 \\
\hline$G_{n}\left(\mathrm{MeV}_{\mathrm{fm}}{ }^{3}\right)$ & -829.99300 & -829.32779 & -826.75466 & -825.97419 & -820.16793 & -820.80162 & -818.64718 & -818.82887 \\
\hline$G_{p}\left(\mathrm{MeV} \cdot \mathrm{fm}^{3}\right)$ & -770.15586 & -769.51970 & -769.92950 & -769.75826 & -771.71259 & -771.63793 & -772.34643 & -773.77497 \\
\hline
\end{tabular}




\section{Results}

In this section, we present the results for the nuclear matter properties in relation to the electric and magnetic dipole excitation properties using the new family of pointcoupling functionals spanning the range of values for the symmetry energy at saturation $J=29,30, \ldots, 36 \mathrm{MeV}$. Thus, we can analyze the effects of varying the symmetry energy in a systematic way.

\subsection{Nuclear Matter Properties and Equation of State}

In Table 3, the nuclear matter properties at saturation density are listed for the family of point-coupling functionals introduced in this work, DD-PC-J29, .., DD-PC-J36, including energy per nucleon $E / A$; incompressibility modulus $K_{0}$, spanning the range of values of the symmetry energy $J=29,30, \ldots, 36 \mathrm{MeV}$; and the slope of the symmetry energy at saturation density $L$. In addition, the respective values are also shown for the DD-PC1 [4] and DD-PCX [26] functionals. Except for the DD-PCX functional, the incompressibility parameters $K_{0}$ is limited to the same value $K_{0}=230 \mathrm{MeV}$. As mentioned above, the DDPCX functional was optimized using the isoscalar monopole resonance energy of ${ }^{208} \mathrm{~Pb}$ to constrain the value of $K_{0}$, which in turn leads to a lower incompressibility parameter around the saturation densities. Because of the strong correlation between the $J$ and $L$ values [49], it is also seen that the $L$ values increase with increasing $J$ values for the DD-PC family of interactions.

Table 3. The nuclear matter saturation properties for the DD-PC family of interactions spanning the range of symmetry energy $J$ and $L$ values. In addition, the properties for the DD-PC1 [4] and DD-PCX [26] functionals are shown.

\begin{tabular}{ccccc}
\hline & E/A $\mathbf{~ ( M e V )}$ & $\boldsymbol{K}_{\mathbf{0}} \mathbf{( M e V )}$ & $J \mathbf{( M e V )}$ & $L \mathbf{~ M e V )}$ \\
\hline DD-PC-J29 & -16.019 & 230.0 & 29.0 & 29.0 \\
DD-PC-J30 & -16.043 & 230.0 & 30.0 & 35.6 \\
DD-PC-J31 & -16.055 & 230.0 & 31.0 & 43.8 \\
DD-PC-J32 & -16.067 & 230.0 & 32.0 & 52.3 \\
DD-PC-J33 & -16.076 & 230.0 & 33.0 & 62.0 \\
DD-PC-J34 & -16.087 & 230.0 & 34.0 & 72.1 \\
DD-PC-J35 & -16.096 & 230.0 & 35.0 & 83.2 \\
DD-PC-J36 & -16.123 & 230.0 & 36.0 & 94.1 \\
DD-PC1 & -16.061 & 230.0 & 33.0 & 70.0 \\
DD-PCX & -16.026 & 213.0 & 31.1 & 46.3 \\
\hline
\end{tabular}

In Figure 1, the EoS of symmetric nuclear matter (panels (a) and (c)) and pure neutron matter (panels (b) and (d)) are displayed for the introduced family of point-coupling functionals together with the ones for the DD-PC1 and DD-PCX interactions. Considering Figure 1a, the results are the same for the densities below $0.3 \mathrm{fm}^{-3}$ for symmetric nuclear matter. Only when moving toward higher densities, differences are obtained in the values of the energy per nucleon. The divergence of the nuclear matter EoS at high-density regions, obtained when using different functionals, is a well-known problem in nuclear physics. Although some probes have been found to constrain this region $[1,17,50]$, the model predictions are quite different and the high-density behavior of the EoS still remains unknown. It is also seen that the DD-PC functionals, which are obtained by varying the symmetry energy parameters, give the same results for the symmetric nuclear matter at each density. In other words, the EoS of symmetric nuclear matter is not sensitive to the changes only in the symmetry energy parameters of the DD-PC functionals. In Figure 1c, the results are presented up to twice the nuclear saturation density $0.34 \mathrm{fm}^{-3}$, and we also compare our results with the order-by-order chiral effective-field theory ( $\chi \mathrm{EFT})$ predictions, with $\mathrm{NN}$ and $3 \mathrm{~N}$ interactions up to $\mathrm{N}^{3} \mathrm{LO}$ (see Reference [51] for more details). Considering the $\mathrm{LO}$ and NLO predictions for the symmetric nuclear matter, binding energy per nucleon $E$ / $A$ decreases smoothly with increasing density, i.e., their behavior is not compatible 
with all other model predictions. However, the behavior of the EoS with the higher-order predictions of the $\chi \mathrm{EFT}$ theory, i.e, $\mathrm{N}^{2} \mathrm{LO}$ and especially $\mathrm{N}^{3} \mathrm{LO}$, appears in qualitative agreement with the DD-PC results.

The results for the energy per nucleon of pure neutron matter are presented in Figure $1 b$,d. In contrast to the symmetric nuclear matter, neutron matter appears rather sensitive to the choice of symmetry energy parameters for the DD-PC family of functionals. As it is known, the symmetry energy is stiffer (softer) for functionals with larger (lower) values of $J$ and $L$. Increasing the value of the symmetry energy at saturation $J$, the energy per nucleon value increases at a faster rate at higher densities, as expected. In Figure 1d, we also compare our results for the pure neutron matter with those from the $\chi \mathrm{EFT}$ theory. The respective EoS of the DD-PC functionals are found to be in agreement with the $\mathrm{N}^{2} \mathrm{LO}$ and $\mathrm{N}^{3} \mathrm{LO}$ results. In particular, the DD-PCX functional appears in remarkable agreement with the $\mathrm{N}^{3} \mathrm{LO}$ results. Among the family of DD-PC functionals, the DD-PC-J31 fits best to the $\mathrm{N}^{3} \mathrm{LO}$ neutron matter EoS.
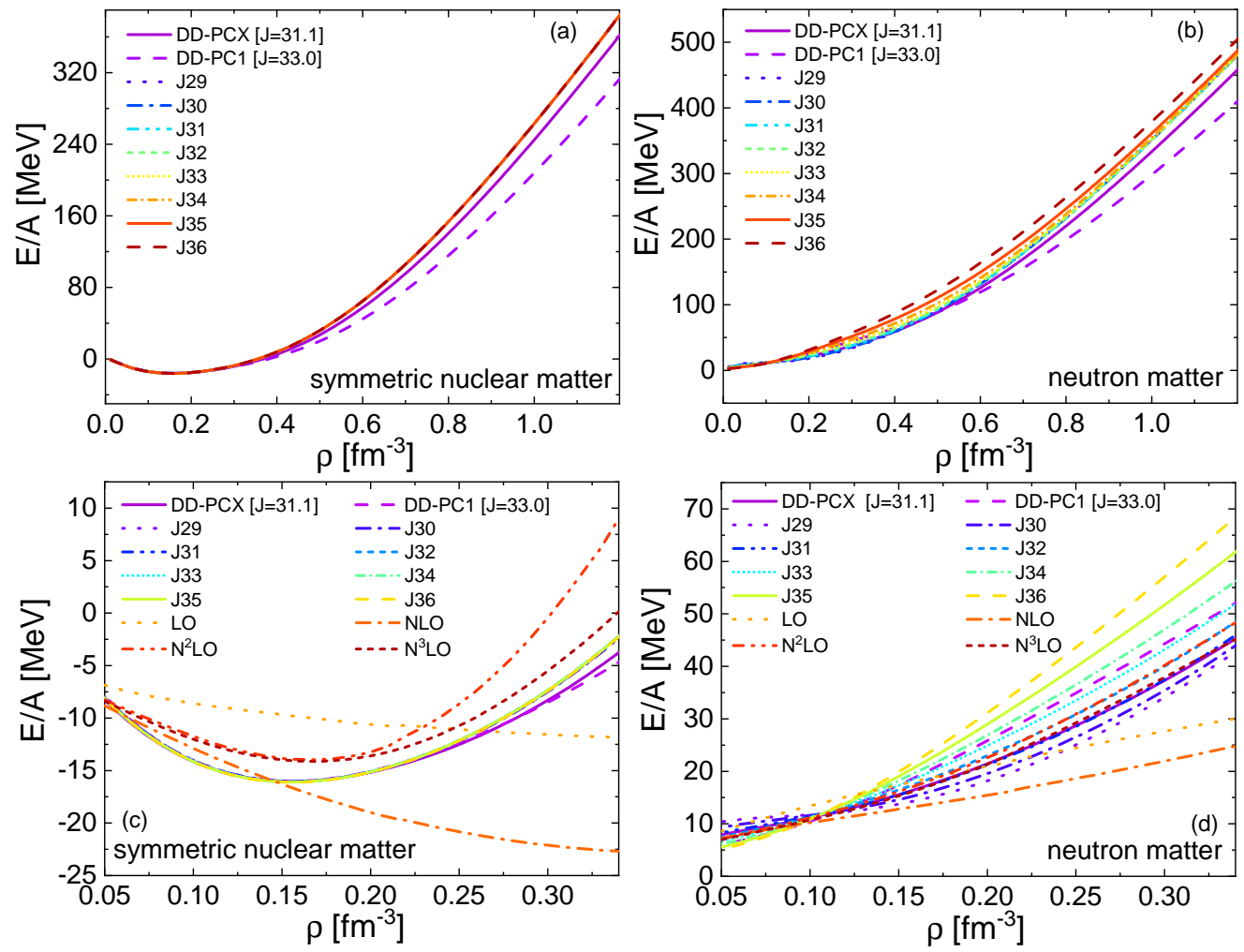

Figure 1. Equation of state of symmetric nuclear matter (a) and pure neutron matter (b) using the family of point-coupling functionals spanning the range of symmetry energy at saturation $J=29-36 \mathrm{MeV}$. The results are also presented using the DD-PC1 and DD-PCX functionals, and the symmetry energy values of the functionals are given within the square brackets in units of $\mathrm{MeV}$. Panels (c,d) are the same, but the results are displayed up to the density $0.34 \mathrm{fm}^{-3}$ to compare with the predictions from chiral effective field theory ( $\chi \mathrm{EFT}$ ) (see Reference [51]).

The dependence of the symmetry energy on the density is presented in Figure 2a, for the same family of point-coupling functionals with $J=29,30, \ldots, 36 \mathrm{MeV}$ alongside the DD-PCX and DD-PC1 functionals. Below the saturation density, the symmetry energy values do not change around $\rho_{0} \approx 0.1 \mathrm{fm}^{-3}$; the results are the same for all the functionals. By increasing the density, the symmetry energy values also start to change in accordance with their different values for $J$ and $L$. The symmetry energy as a function of density is higher (lower) for the functionals with larger (lower) values of the symmetry energy parameters, as expected [10]. In Figure 2b, we also display the symmetry energy of other models at lower densities to compare our results. Apart from the $\chi$ EFT theory results (LO, NLO, $\mathrm{N}^{2} \mathrm{LO}$, and $\mathrm{N}^{3} \mathrm{LO}$ ), we also present the symmetry energy limits obtained using the Isobaric 
analog state (IAS) and neutron skin $\left(\Delta R_{n p}\right)$ constraints (see Reference [22]). It is known that the IAS can be used to constrain the isovector channel of the nuclear energy density functionals. In this work, we find that almost all models predict increasing symmetry energy with increasing density, except for $\mathrm{N}^{2} \mathrm{LO}$, which decreases slightly after $\rho>0.25 \mathrm{fm}^{-3}$. We find that the predictions and behavior of the symmetry energy using the point-coupling DD-PCX and DD-PC-J31 functionals are in good agreement with the results of the NLO and $\mathrm{N}^{3} \mathrm{LO}$. Using the $\chi$ EFT theory, the symmetry energy and its slope was constrained to $J=31.7 \pm 1.1 \mathrm{MeV}$ and $L=59.8 \pm 4.1 \mathrm{MeV}$ around the nuclear saturation density [51]. Moreover, the symmetry energy was constrained between $0.04 \leq \rho \leq 0.13 \mathrm{fm}^{-3}$ using the IAS $+\Delta R_{n p}$ information, and the $J$ and $L$ values were obtained between 30.2-33.7 MeV and 35-70 MeV around the saturation densities [22], respectively. Considering the predictions in the low density region and around the saturation densities, we find that point-coupling DD-PCX $(J=31.12 \pm 0.32 \mathrm{MeV})$ and DD-PC-J31 functionals appear very compatible with the results from the $\chi$ EFT theory and the IAS $+\Delta R_{n p}$ constraints on the symmetry energy. In the following section the family of DD-PC functionals will also be tested with the dipole polarizability data on finite nuclei.
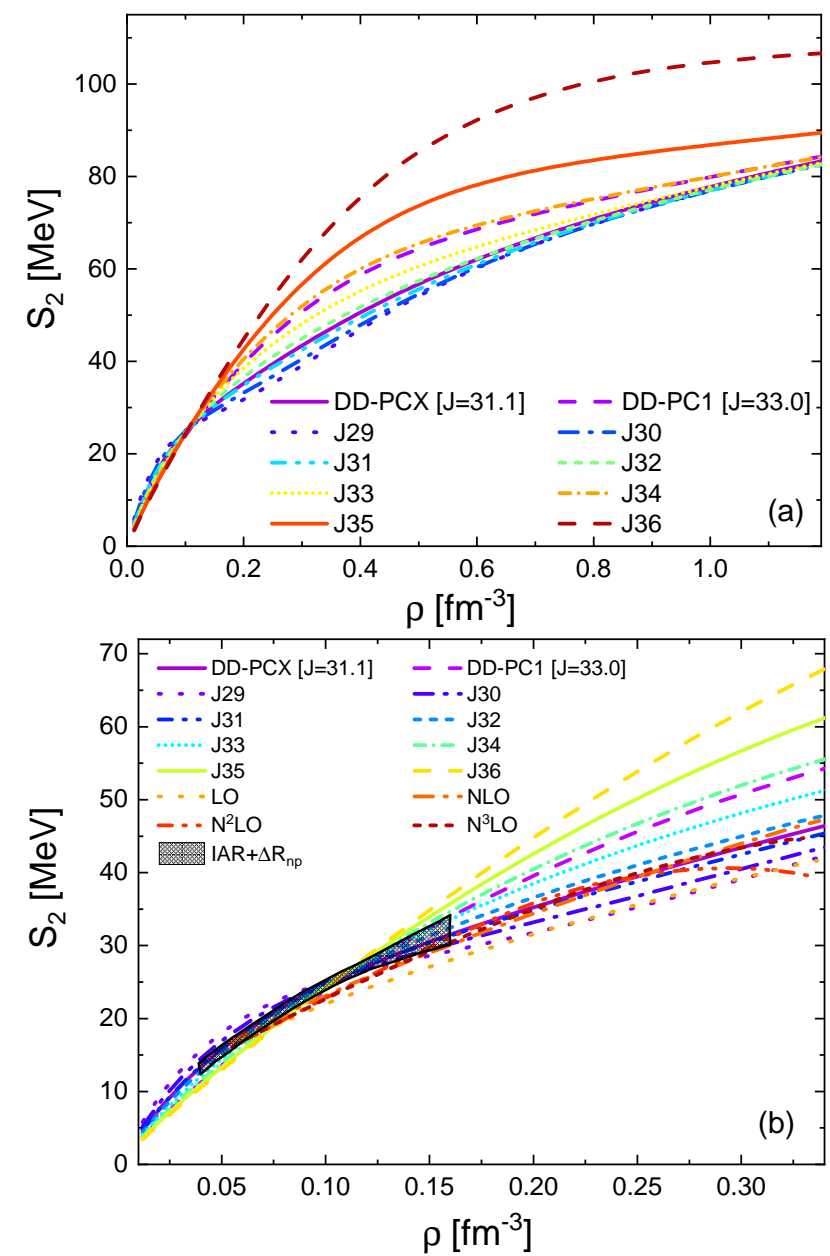

Figure 2. (a) Symmetry energy as a function of density using a family of point-coupling functionals with $J=29,30, \ldots, 36 \mathrm{MeV}$ alongside the DD-PC1 and DD-PCX functionals. For the DD-PCX and DD-PC1 functionals, the symmetry energy values around the saturation densities are given within the square brackets in units of $\mathrm{MeV}$. (b) The same but for densities up to $0.34 \mathrm{fm}^{-3}$, with the results of the chiral effective field theory ( $\chi \mathrm{EFT}$ ) (see Reference [51]). The isobaric analog state (IAS) and neutron skin $\left(\Delta R_{n p}\right)$ constraints on the symmetry energy (see Reference [22] for more details) are also presented. 


\subsection{Dipole Polarizability and Neutron Skin}

The dipole polarizability $\alpha_{D}$ was recently established as a useful additional constraint for the isovector channel in EDFs [21]. It is defined as

$$
\alpha_{D}=\frac{8 \pi e^{2}}{9} \int_{0}^{\infty} E^{-1} S_{E 1}(E) d E=\frac{8 \pi e^{2}}{9} m_{-1}(E 1),
$$

where $E$ is the excitation energy and $m_{-1}(E 1)$ is the inverse energy weight sum of the isovector dipole transition strength. The strength function is given by

$$
S_{E 1}(E)=\sum_{i}\left|\left\langle\omega_{i}\left|\hat{\mathcal{Q}}^{(\mathrm{IV}-\mathrm{E} 1)}\right| \Phi\right\rangle\right|^{2} \delta\left(\hbar \omega_{i}-E\right)
$$

where $|\Phi\rangle$ denotes the ground-state of nucleus and $\hat{\mathcal{Q}}^{(\mathrm{IV}-\mathrm{E} 1)}$ is the isovector transition dipole operator [27]. The transition matrix elements can be calculated using the quasiparticle random phase approximation [27,52].

In numerous studies both relativistic and nonrelativistic EDFs have been tested using the experimental data on $\alpha_{D}$ for ${ }^{48} \mathrm{Ca},{ }^{68} \mathrm{Ni},{ }^{120} \mathrm{Sn}$, and ${ }^{208} \mathrm{~Pb}$ (see [10] and the references therein). Another useful isovector quantity to be considered in probing the isovector sector of the EDFs is the neutron-skin thickness [49]. However, its data are still rather limited, often model dependent, or have large uncertainties such as in the case of parity violating electron scattering experiment (PREX) [19]. Therefore, for any novel functional, it is interesting to explore both the dipole polarizability and neutron-skin thickness, and when possible, to compare the results of model calculations with the experimental data.

We start our analysis with the neutron-skin thickness $\Delta R_{n p}$ for the point-coupling functionals DD-PC1 and DD-PCX. The neutron skin thickness of a nucleus is defined as $\Delta R_{n p}=\left\langle r_{n}^{2}\right\rangle^{1 / 2}-\left\langle r_{p}^{2}\right\rangle^{1 / 2}$, and $\left\langle r_{n}^{2}\right\rangle^{1 / 2}$ and $\left\langle r_{p}^{2}\right\rangle^{1 / 2}$ represent the neutron and proton root-mean-square radii, respectively. In Figure 3, we display the available results from antiprotonic atom experiments $[53,54]$ along with the DD-PC1 and DD-PCX results. We also display the experimental and theoretical results for the Sn chain in Figure 4. As can be seen in Figure 3, the experimental data have large error bars for most of the nuclei. Comparing the experimental results with the DD-PC1 and DD-PCX ones, it is seen that both functionals provide reasonable results and that the predictions are generally within the experimental error bars. It is known that the symmetry energy $(J)$ and slope of the symmetry energy $(L)$ at saturation are strongly correlated with the neutron skin thickness in finite nuclei [49]. Since the $J$ and $L$ values are lower for the DD-PCX functional than it is for DD-PC1, in the former case, smaller neutron skin radii are predicted for all nuclei, as expected.

As discussed above, the symmetry energy parameters are strongly correlated with the dipole polarizability as well as with the neutron skin thickness of nuclei. In Figure 5, we display the relationship between the dipole polarizability and neutron skin thickness results using the previously introduced family of point-coupling functionals obtained for ${ }^{48} \mathrm{Ca},{ }^{68} \mathrm{Ni},{ }^{120} \mathrm{Sn}$ and ${ }^{208} \mathrm{~Pb}$. An increase in the symmetry energy parameters leads to an increase in the neutron skin thickness of nuclei. As shown in figure, by increasing the $J$ value of the functional, both the neutron thickness and dipole polarizability predictions increase linearly. The horizontal violet bands represent the measured dipole polarizability values of nuclei with error bars from References [55-60]. The vertical green bands denote the neutron skin thickness values obtained from model-averaged results (see Reference [60]). The overlap between the two bands defines an area denoted by red rectangle, which restricts the original family of DD-PC interactions to those that can simultaneously describe the dipole polarizability and neutron-skin thickness predictions. For the nuclei considered in the analysis, the results of the DD-PC functional with $J=31 \mathrm{MeV}$ are in good agreement with all the predictions for $\alpha_{D}$ and $\Delta R_{n p}$. In addition, we can observe that the DD-PCX interaction for all nuclei fits into the restricted range of $\alpha_{D}$ and $\Delta R_{n p}$ values. As can be seen 
from Figure 5, the DD-PC1 functional predicts higher values for dipole polarizability and neutron skin thickness compared to DD-PCX. In addition, the DD-PC1 results are outside of the ranges given by the experiment and model calculations.

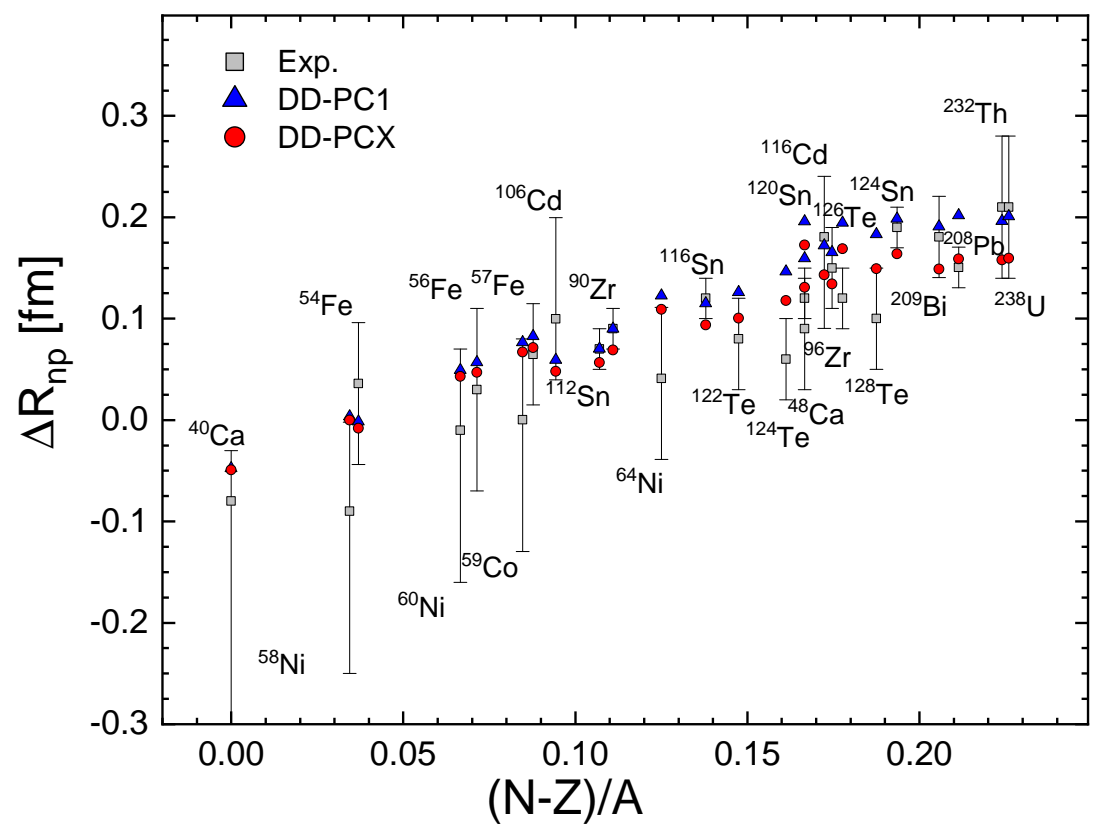

Figure 3. The neutron skin thickness as a function of the neutron excess for the DD-PC1 and DDPCX functionals. The experimental data are taken from References [53,54] in which the proton distributions were obtained from electron scattering data for Sn nuclei [61] and from muonic atom data for others [62-64].

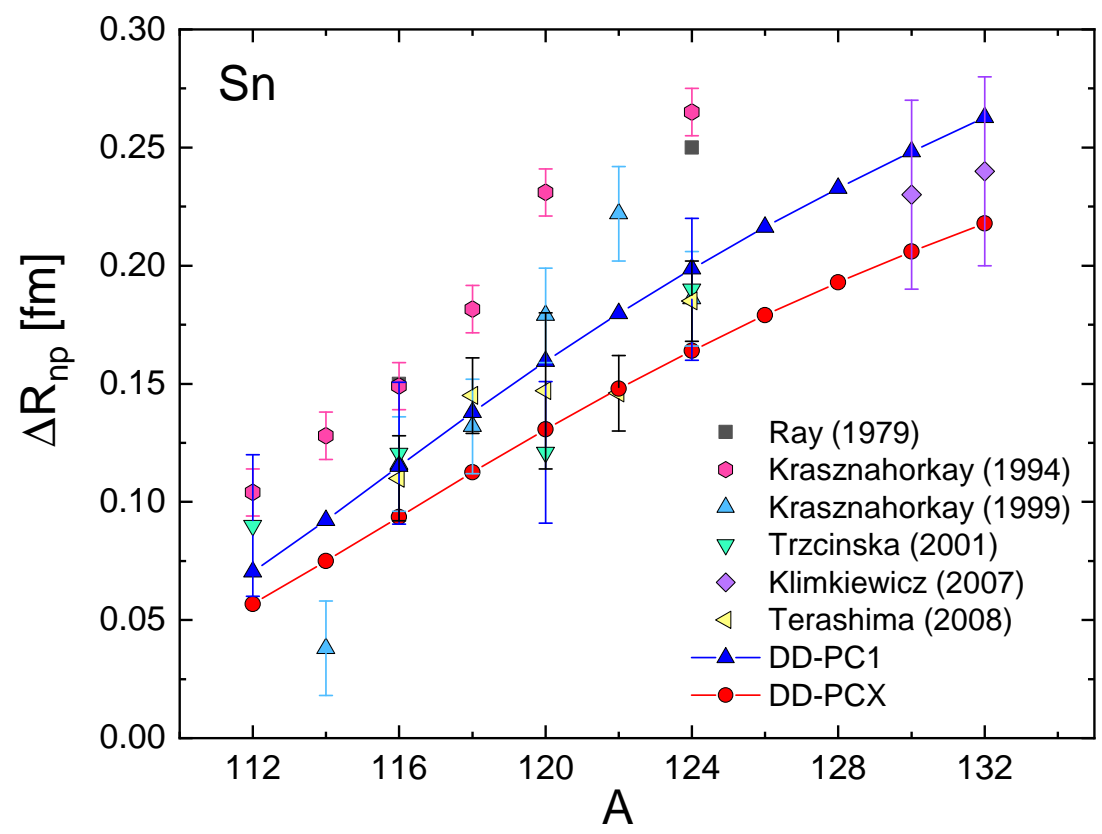

Figure 4. The neutron skin thickness for $\mathrm{Sn}$ isotopes as a function of the mass number. The experimental data are taken from References [53,65-69]. 

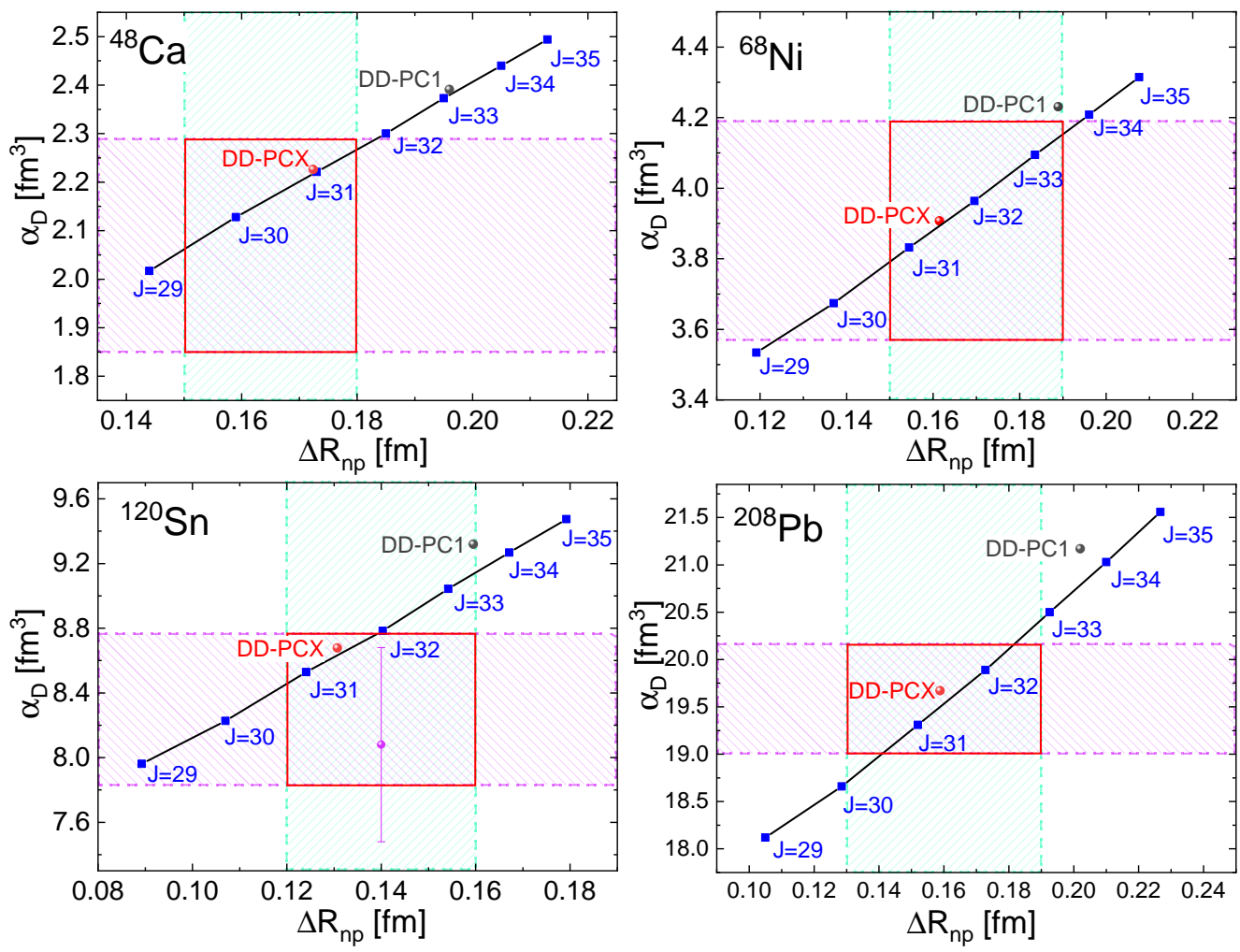

Figure 5. Dipole polarizability as a function of neutron skin thickness for ${ }^{48} \mathrm{Ca},{ }^{68} \mathrm{Ni},{ }^{120} \mathrm{Sn}$ and ${ }^{208} \mathrm{~Pb}$ using the relativistic point-coupling functionals. The experimental data for the dipole polarizability values are taken from References [55-60]. The recent experimental data for ${ }^{120} \mathrm{Sn}$ is taken from Reference [58] and displayed $\left(\alpha_{D}=8.08 \pm 0.6 \mathrm{fm}^{3}\right)$ with the violet shaded sphere. The band for the neutron radii represents the model-averaged results using many functionals (see Reference [60] for more information).

Since the DD-PCX is optimized using the $\alpha_{D}$ data for ${ }^{208} \mathrm{~Pb}$, the results for this nucleus are found in the middle of the intersection point of the experimental data for dipole polarizability and model-averaged results for neutron skin thickness. Although we only used the dipole polarizability of ${ }^{208} \mathrm{~Pb}$ in the optimization of the DD-PCX functional, it is clear that it can successfully predict the dipole polarizabilities and neutron-skin thicknesses of other nuclei. The predictions of the DD-PCX for $\alpha_{D}$ and $\Delta R_{n p}$ also indicate that the isovector channel of the respective relativistic EDF is constrained successfully.

In Figure 6, the dipole polarizability results of the ${ }^{208} \mathrm{~Pb}$ is presented along with the results of the ${ }^{48} \mathrm{Ca},{ }^{68} \mathrm{Ni}$, and ${ }^{120} \mathrm{Sn}$. We present our calculations using the DD-PC family of functionals varying the $J$ value as well as the DD-PC1 and DD-PCX ones, and the theoretical results using the other functionals were taken from Reference [60]. The ranges of experimental data are also denoted with vertical and horizontal bands for the considered nuclei. The band overlap area, denoted by the red line, reduces a large set of nonrelativistic and relativistic functionals to only a few that successfully reproduce the experimental data on $\alpha_{D}$ for all nuclei under consideration. The most reliable functionals that can predict the dipole polarizability of all nuclei are the DD-PC functional with $J=31 \mathrm{MeV}$ and the DD-PCX functional. The results show that the approach based on construction of a family of point-coupling interactions spanning the range of values of $J$ and its restriction by the $\alpha_{D}$ data provides a similar constraint on the symmetry energy as that of the DD-PCX functional obtained by including $\alpha_{D}$ value for ${ }^{208} \mathrm{~Pb}$ directly in minimizing the $\chi^{2}$ function to determine the model parameters. Both strategies result in successful optimization of the isovector channel of the relativistic EDF. 

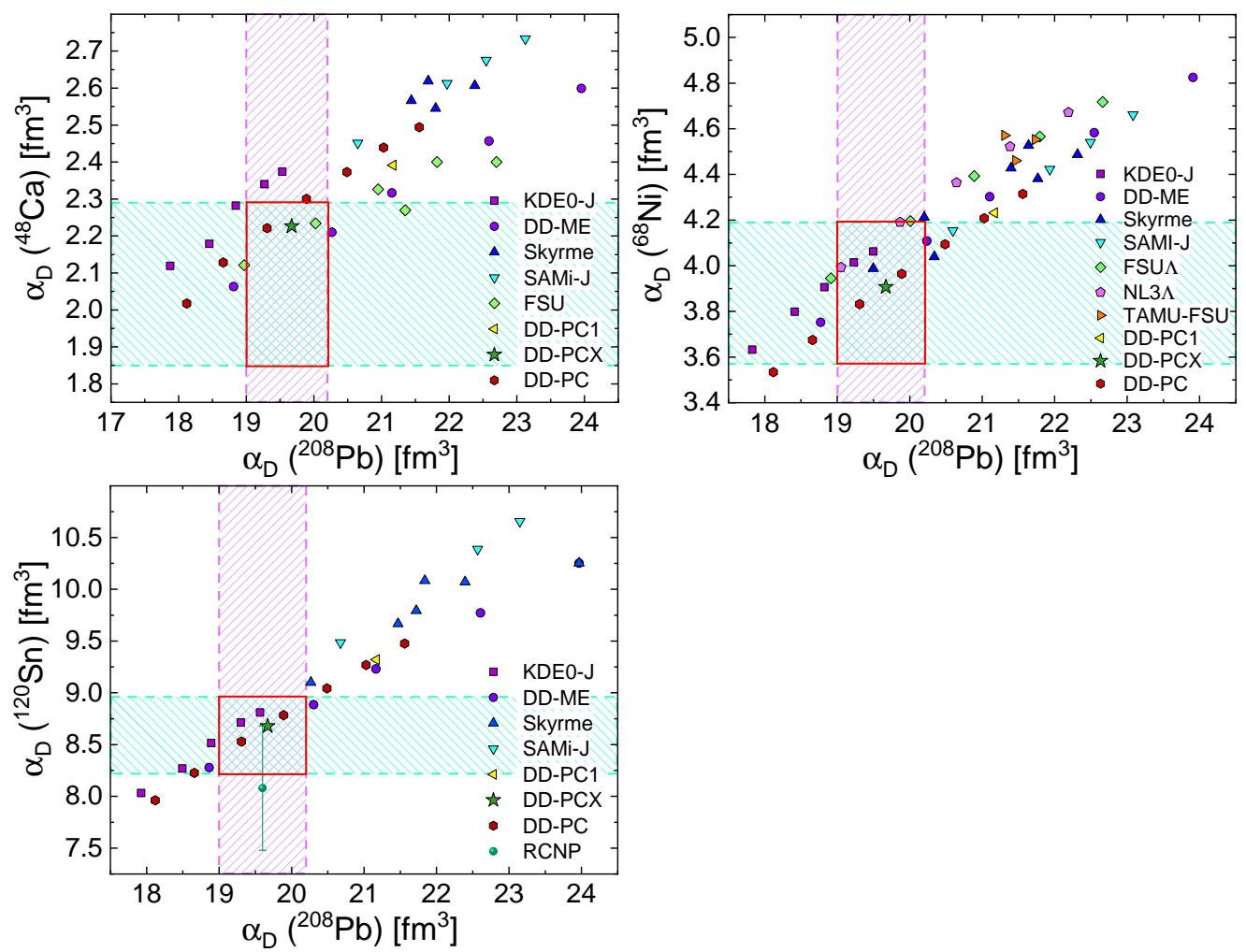

Figure 6. Comparison of the theoretical results for the dipole polarizability with the experimental data for ${ }^{48} \mathrm{Ca}-{ }^{208} \mathrm{~Pb},{ }^{68} \mathrm{Ni}^{2}{ }^{208} \mathrm{~Pb}$, and ${ }^{120} \mathrm{Sn}-{ }^{208} \mathrm{~Pb}$. The theoretical results for the KDE0-J, DD-ME, Skyrme, SAMI-J, NL3 $\Lambda$, and FSU functionals are taken from Reference [60]. The experimental data with the error bars are shown as the shaded regions over the graph. The experimental data for ${ }^{48} \mathrm{Ca}\left(\alpha_{D}=2.07 \pm 0.22 \mathrm{fm}^{3}\right),{ }^{68} \mathrm{Ni}\left(\alpha_{D}=3.88 \pm 0.31 \mathrm{fm}^{3}\right)$, and ${ }^{208} \mathrm{~Pb}\left(\alpha_{D}=19.6 \pm 0.6 \mathrm{fm}^{3}\right)$ are taken from References [55], [56,60], and [59,60], respectively. For ${ }^{120} \mathrm{Sn}$, the experimental value from References [57,60] $\left(\alpha_{D}=8.59 \pm 0.37 \mathrm{fm}^{3}\right)$ is also shown using the shaded regions, and the recent data from Reference [58] $\left(\alpha_{D}=8.08 \pm 0.6 \mathrm{fm}^{3}\right)$ is displayed with a green shaded sphere.

\subsection{Sensitivity of Magnetic-Dipole Excitation to Symmetry Energy}

In the following, we investigate the sensitivity of magnetic dipole (M1) excitation properties on the symmetry energy parameters by using the same family of the relativistic point-coupling functionals as discussed in previous sections. Thus, the RRPA calculations for the isovector (IV) M1 excitations in ${ }^{48} \mathrm{Ca}$ and ${ }^{208} \mathrm{~Pb}$ nuclei are performed by using the DD-PC functionals with $J=29-36 \mathrm{MeV}$. In References [36,70], the relativistic EDF with point-coupling interaction has been for the first time employed in the description of IV-M1 excitations by employing the RHB $+\mathrm{R}(\mathrm{Q}) \mathrm{RPA}$ model. The same framework and model parameters from Reference [36] were used in the present study, except that its DD-PC parameterization was replaced to account for different values of the symmetry energy at the saturation density, $J \equiv S\left(\rho=\rho_{0}\right)$. In the case of ${ }^{48} \mathrm{Ca}$ and ${ }^{208} \mathrm{~Pb}$, our quasiparticle random phase approximation (QRPA) method reduces to the normal Random Phase Approximation (RPA).

Up to the one-body-RPA level, the IV-M1 operator, which depends on the spin $\hat{s}_{v}$ and orbital angular momentum $\hat{l}_{v}$, is given as

$$
\hat{\mathcal{Q}}_{v}^{(\mathrm{IV}-\mathrm{M} 1)}=\sqrt{\frac{3}{4 \pi}} \mu_{\mathrm{N}} \sum_{k \in A}\left(g_{l}^{(\mathrm{IV})} \hat{l}_{v}(k)+g_{s}^{(\mathrm{IV})} \hat{s}_{v}(k)\right) \hat{\tau}_{3}(k),
$$

where $\mu_{\mathrm{N}}$ is the nuclear magneton, $\hat{\tau}_{3}=1(-1)$ for protons (neutrons), and $g$ coefficients are given as $g_{l}^{(\mathrm{IV})}=0.5$ and $g_{s}^{(\mathrm{IV})}=4.706[71,72]$. For simplicity, we neglect the quenching effect in $g$ coefficients [73-75]. Then, the M1-excitation strength is computed as 


$$
\frac{d B_{\mathrm{M} 1}}{d E}=\sum_{i} \delta\left(E-\hbar \omega_{i}\right) \sum_{v}\left|\left\langle\omega_{i}\left|\hat{\mathcal{Q}}_{v}^{(\mathrm{IV}-\mathrm{M} 1)}\right| \Phi\right\rangle\right|^{2}
$$

For demonstration purposes, instead of $\delta\left(E-\hbar \omega_{i}\right)$, we smear the strength with a Cauchy-Lorenz profile, for which the full width at half maximum (FWHM) is $1.0 \mathrm{MeV}$.

In Figure 7, the transition strength distributions for M1 excitations are presented for the family of DD-PC interactions spanning the symmetry energy in the range $J=29-36 \mathrm{MeV}$. One can observe a systematic dependence of the M1 excitation energy on the $J$ value of the effective interaction used in the calculation. Namely, the small (large) symmetry energy coincides with the high (low) excitation energy obtained for the M1 transition. However, when considering the corresponding transition strengths, no remarkable change is obtained among $J=29-36$ results.
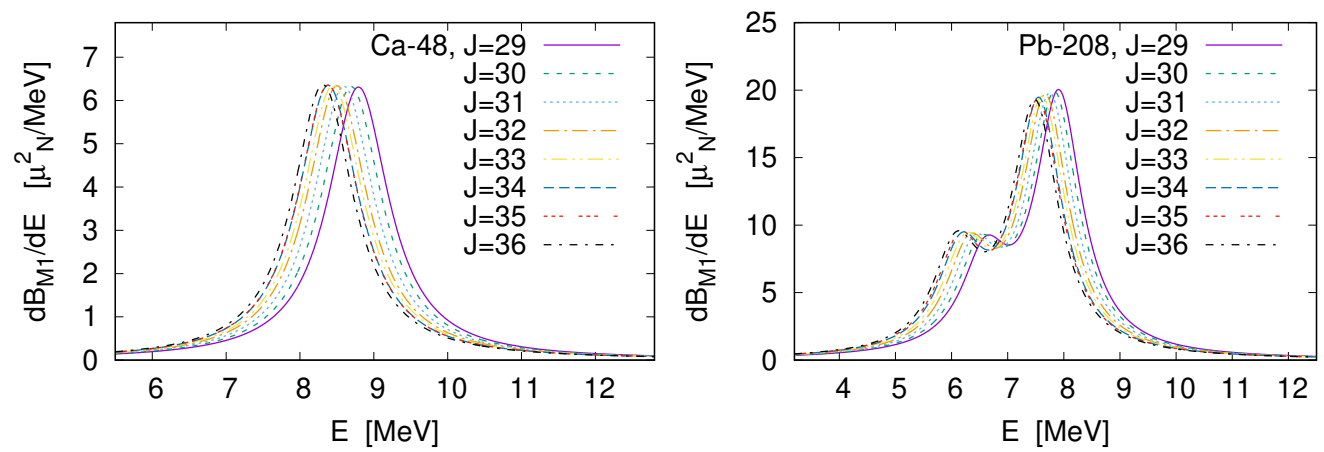

Figure 7. The M1 transition strength distribution for ${ }^{48} \mathrm{Ca}$ and ${ }^{208} \mathrm{~Pb}$ obtained by the family of DD-PC interactions with the symmetry energy at the saturation, $J=29,30, \ldots, 36 \mathrm{MeV}$.

Next, we consider the moments of M1 transitions, that is,

$$
m_{K}(\mathrm{M} 1) \equiv \int E^{K} \frac{d B_{\mathrm{M} 1}}{d E} d E,
$$

where $K=0$ and $K=1$ indicate the non-energy-weighted and energy-weighted sum of the B (M1) transition strength, respectively. Similar to that in Section 3.2, here, we consider the relationship between the M1 excitation properties and the neutron-skin thickness for the family of DD-PC interactions. In Figure 8, the energy-weighted sum of B (M1) strength for ${ }^{48} \mathrm{Ca}$ is presented as a function of the neutron-skin thickness $\Delta R_{\mathrm{np}}$, obtained by using the DD-PC functionals spanning the range of $J$ values as denoted in the figure. In the lower panel, the dependence of the M1 transition centroid energy on $\Delta R_{\mathrm{np}}$ is shown. Figure 9 shows the same analysis but for ${ }^{208} \mathrm{~Pb}$. Both for the ${ }^{48} \mathrm{Ca}$ and ${ }^{208} \mathrm{~Pb}$ nuclei, the $m_{1}(\mathrm{M} 1)$ value becomes reduced (enhanced) for the larger (smaller) neutron-skin thickness $\Delta R_{\mathrm{np}}$ or equivalently larger (smaller) $J$ value. This is consistent with the sensitivity found in Figure 7. Thus, the M1 transitions may also be used as an additional constraint for the symmetry energy properties. Notice also that, for the ${ }^{208} \mathrm{~Pb}$ nucleus, since the $d B_{\mathrm{M} 1} / d E$ distribution includes two major peaks, its $m_{1}(\mathrm{M} 1)$ value does not simply indicate the major excitation energy, in contrast to the ${ }^{48} \mathrm{Ca}$ nucleus. For the non-energy-weighted sum of the M1 strength $m_{0}(\mathrm{M} 1)$, no significant sensitivity to $J$ is obtained. Therefore, as shown in Figure 8, the centroid energy, $\bar{E} \equiv m_{1}(\mathrm{M} 1) / m_{0}(\mathrm{M} 1)$, displays the same tendency as the $m_{1}$ (M1) value. This constant $m_{0}$ (M1) value is in agreement with previous studies [70,76], where the non-energy-weighted sum rule does not change for the M1 mode in considering the case when the pairing correlation is negligible. 


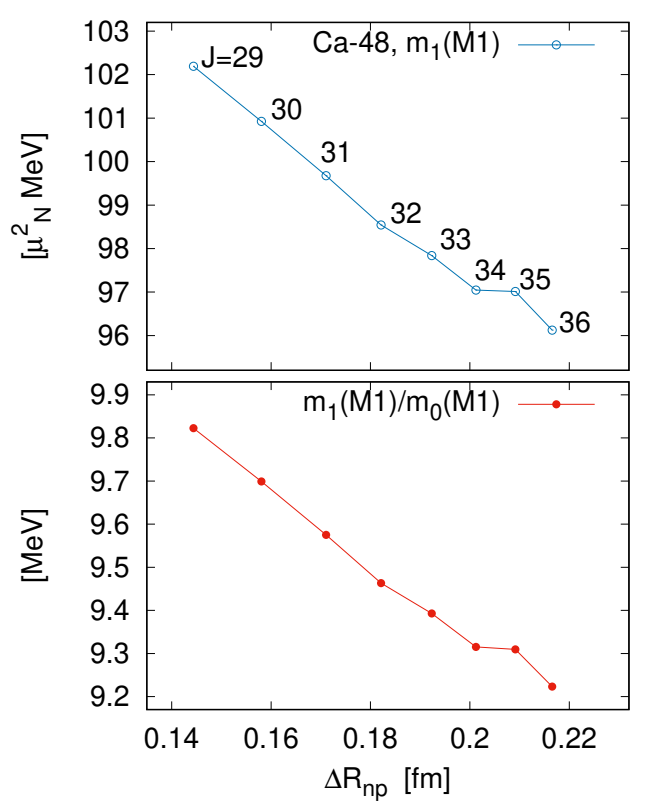

Figure 8. (Top) Energy-weighted M1-summation results for ${ }^{48} \mathrm{Ca}$ obtained with the present DD-PC interactions. (Bottom) Centroid energy of M1 transition, $\bar{E} \equiv m_{1}(\mathrm{M} 1) / m_{0}(\mathrm{M} 1)$.

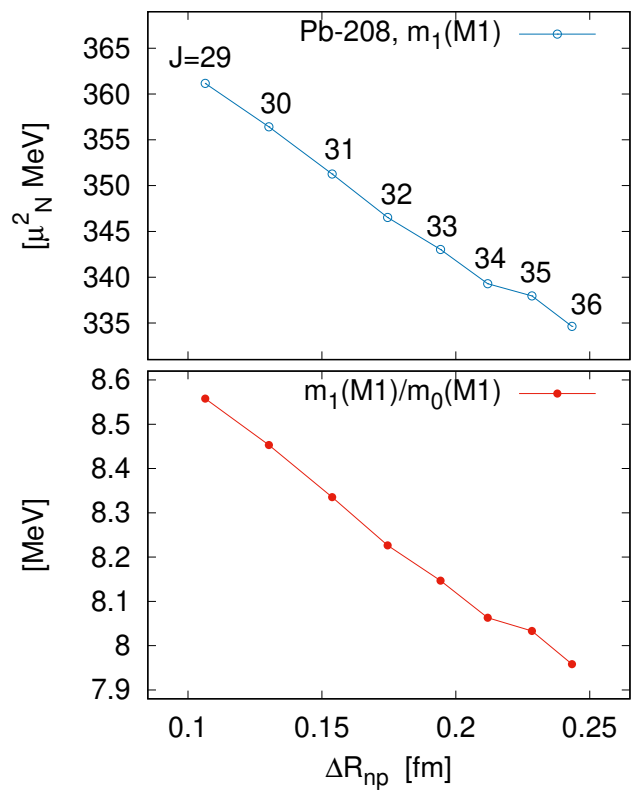

Figure 9. The same as Figure 8 but for ${ }^{208} \mathrm{~Pb}$.

We note that, in this work, we performed the first investigation of the relation between M1 excitation and symmetry energy parameters. On the other hand, for further applications, there remain several tasks. Especially in theoretical calculations, the isovectorpseudovector (IV-PV) interaction plays an important role in the description of M1-excitation energy [36,70]. Its coupling parameter, however, still has a finite ambiguity $[36,70]$. Because of this ambiguity, the present RPA calculations have not completely reproduced the experimental data on $\mathrm{M} 1$ centroid energies, that is, $\bar{E}_{\text {exp. }}=10.2 \mathrm{MeV}$ for ${ }^{48} \mathrm{Ca}$ [77-80] and $\bar{E}_{\text {exp. }}=7.3 \mathrm{MeV}$ for ${ }^{208} \mathrm{~Pb}$ [81]. We are planning more systematic optimization of theoretical parameters to remedy this gap. Nevertheless, we emphasize that the results of the present analysis display the sensitivity of M1 excitations to symmetry energy at saturation density. 


\title{
4. Conclusions
}

Constraining the isovector channel of the EDFs, which is of paramount importance for description of the symmetry energy of the nuclear EoS, represents a continuous challenge for the nuclear physics and astrophysics community. In this work, we investigated the possible constraints to symmetry energy by exploiting the properties of dipole electric and magnetic excitations in finite nuclei. For this purpose, a novel family of 8 relativistic densitydependent point-coupling interactions is established, which varies the symmetry energy at saturation in the range of values $J=29-36 \mathrm{MeV}$. We exploited this family of functionals in the analysis of possible isovector constraints, in particular, the dipole polarizability and magnetic dipole excitation energy in nuclei. It is shown that, in this way, the symmetry energy can be further restricted by using available experimental data on electric and magnetic excitation properties. Through this analysis, we also validated the isovector properties of the DD-PCX functional, which was recently established not only using the nuclear ground-state properties of nuclei but also using additional constraints from the experimental data on dipole polarizability and isoscalar monopole resonance energy in ${ }^{208} \mathrm{~Pb}$ directly in $\chi^{2}$ optimization. The DD-PCX functional, together with the family of DDPC functionals introduced in this work that cover a range of values for the symmetry energy at the saturation density, represent useful tools for further studies necessary to constrain the properties of nuclear EoS. The new set of functionals also provides an interesting possibility to establish EoS for supernovae and compact stars [2] in order to explore the sensitivity of their properties on variations in the EoS in a systematic way. Available information on the neutron star mass-radius relationship can also provide useful constraints on the DD-PC functionals.

Author Contributions: The authors have contributed to this work as follows. Conceptualization, E.Y. and N.P.; methodology, N.P., E.Y., and T.O.; formal analysis, E.Y. and T.O.; investigation, E.Y. and T.O.; writing-original draft preparation, E.Y. and T.O.; writing—review and editing, N.P.; visualization, E.Y. and T.O.; funding acquisition, N.P. All authors have read and agreed to the published version of the manuscript.

Funding: This work is supported by the Croatian Science Foundation under the project Structure and Dynamics of Exotic Femtosystems (IP-2014-09-9159) and by the QuantiXLie Centre of Excellence, a project co-financed by the Croatian Government and European Union through the European Regional Development Fund, the Competitiveness and Cohesion Operational Programme (KK.01.1.1.01).

Institutional Review Board Statement: Not applicable.

Informed Consent Statement: Not applicable.

Data Availability Statement: Not applicable.

Conflicts of Interest: The authors declare no conflict of interest.

\author{
Abbreviations \\ The following abbreviations are used in this manuscript: \\ EDF Energy density functional \\ RHB Relativistic Hartree-Bogoliubov \\ QRPA Quasiparticle random phase approximation \\ EoS Equation of state
}




\section{References}

1. Lattimer, J.M. The Nuclear Equation of State and Neutron Star Masses. Annu. Rev. Nucl. Part. Sci. 2012, 62, 485-515. [CrossRef]

2. Oertel, M.; Hempel, M.; Klähn, T.; Typel, S. Equations of state for supernovae and compact stars. Rev. Mod. Phys. 2017, 89, 015007. [CrossRef]

3. Lalazissis, G.A.; Nikšić, T.; Vretenar, D.; Ring, P. New relativistic mean-field interaction with density-dependent meson-nucleon couplings. Phys. Rev. C 2005, 71, 024312. [CrossRef]

4. Nikšić, T.; Vretenar, D.; Ring, P. Relativistic nuclear energy density functionals: Adjusting parameters to binding energies. Phys. Rev. C 2008, 78, 034318. [CrossRef]

5. Chen, W.C.; Piekarewicz, J.; Centelles, M. Giant monopole energies from a constrained relativistic mean-field approach. Phys. Rev. C 2013, 88, 024319. [CrossRef]

6. Chen, W.C.; Piekarewicz, J. Building relativistic mean field models for finite nuclei and neutron stars. Phys. Rev. C 2014, 90, 044305. [CrossRef]

7. Kortelainen, M.; Lesinski, T.; Moré, J.; Nazarewicz, W.; Sarich, J.; Schunck, N.; Stoitsov, M.V.; Wild, S. Nuclear energy density optimization. Phys. Rev. C 2010, 82, 024313. [CrossRef]

8. Kortelainen, M.; McDonnell, J.; Nazarewicz, W.; Reinhard, P.G.; Sarich, J.; Schunck, N.; Stoitsov, M.V.; Wild, S.M. Nuclear energy density optimization: Large deformations. Phys. Rev. C 2012, 85, 024304. [CrossRef]

9. Kortelainen, M.; McDonnell, J.; Nazarewicz, W.; Olsen, E.; Reinhard, P.G.; Sarich, J.; Schunck, N.; Wild, S.M.; Davesne, D.; Erler, J.; et al. Nuclear energy density optimization: Shell structure. Phys. Rev. C 2014, 89, 054314. [CrossRef]

10. Roca-Maza, X.; Paar, N. Nuclear equation of state from ground and collective excited state properties of nuclei. Prog. Part. Nucl. Phys. 2018, 101, 96-176. [CrossRef]

11. Lattimer, J.M.; Lim, Y. Constraining the symmetry parameters of the nuclear interaction. Astrophys. J. 2013, 771, 51. [CrossRef]

12. Horowitz, C.J.; Brown, E.F.; Kim, Y.; Lynch, W.G.; Michaels, R.; Ono, A.; Piekarewicz, J.; Tsang, M.B.; Wolter, H.H. A way forward in the study of the symmetry energy: Experiment, theory, and observation. J. Phys. G Nucl. Part. Phys. 2014, 41, 093001. [CrossRef]

13. Tsang, M.B.; Stone, J.R.; Camera, F.; Danielewicz, P.; Gandolfi, S.; Hebeler, K.; Horowitz, C.J.; Lee, J.; Lynch, W.G.; Kohley, Z.; et al. Constraints on the symmetry energy and neutron skins from experiments and theory. Phys. Rev. C 2012, 86, 015803. [CrossRef]

14. Centelles, M.; Roca-Maza, X.; Viñas, X.; Warda, M. Nuclear Symmetry Energy Probed by Neutron Skin Thickness of Nuclei. Phys. Rev. Lett. 2009, 102, 122502. [CrossRef] [PubMed]

15. Garg, U.; Colò, G. The compression-mode giant resonances and nuclear incompressibility. Prog. Part. Nucl. Phys. 2018, 101, 55-95. [CrossRef]

16. Stone, J.R.; Stone, N.J.; Moszkowski, S.A. Incompressibility in finite nuclei and nuclear matter. Phys. Rev. C 2014, 89, 044316. [CrossRef]

17. Danielewicz, P.; Lacey, R.; Lynch, W.G. Determination of the Equation of State of Dense Matter. Science 2002, 298, 1592-1596. [CrossRef]

18. Baldo, M.; Burgio, G. The nuclear symmetry energy. Prog. Part. Nucl. Phys. 2016, 91, 203-258. [CrossRef]

19. Abrahamyan, S.; Ahmed, Z.; Albataineh, H.; Aniol, K.; Armstrong, D.S.; Armstrong, W.; Averett, T.; Babineau, B.; Barbieri, A.; Bellini, V.; et al. Measurement of the Neutron Radius of ${ }^{208} \mathrm{~Pb}$ through Parity Violation in Electron Scattering. Phys. Rev. Lett. 2012, 108, 112502. [CrossRef]

20. Adhikari, D.; Albataineh, H.; Androic, D.; Aniol, K.; Armstrong, D.S.; Averett, T.; Barcus, S.; Bellini, V.; Beminiwattha, R.S.; Benesch, J.F.; et al. An Accurate Determination of the Neutron Skin Thickness of 208Pb through Parity-Violation in Electron Scattering. arXiv 2021, arXiv:2102.10767.

21. Piekarewicz, J.; Agrawal, B.K.; Colò, G.; Nazarewicz, W.; Paar, N.; Reinhard, P.G.; Roca-Maza, X.; Vretenar, D. Electric dipole polarizability and the neutron skin. Phys. Rev. C 2012, 85, 041302. [CrossRef]

22. Danielewicz, P.; Lee, J. Symmetry energy II: Isobaric analog states. Nucl. Phys. A 2014, 922, 1-70.

23. Vretenar, D.; Paar, N.; Nikšić, T.; Ring, P. Spin-Isospin Resonances and the Neutron Skin of Nuclei. Phys. Rev. Lett. 2003, 91, 262502. [CrossRef] [PubMed]

24. Krasznahorkay, A.; Paar, N.; Vretenar, D.; Harakeh, M. Anti-analog giant dipole resonances and the neutron skin of nuclei. Phys. Lett. B 2013, 720, 428-432. [CrossRef]

25. Cao, L.G.; Roca-Maza, X.; Colò, G.; Sagawa, H. Constraints on the neutron skin and symmetry energy from the anti-analog giant dipole resonance in ${ }^{208} \mathrm{~Pb}$. Phys. Rev. C 2015, 92, 034308. [CrossRef]

26. Yüksel, E.; Marketin, T.; Paar, N. Optimizing the relativistic energy density functional with nuclear ground state and collective excitation properties. Phys. Rev. C 2019, 99, 034318. [CrossRef]

27. Paar, N.; Ring, P.; Nikšić, T.; Vretenar, D. Quasiparticle random phase approximation based on the relativistic Hartree-Bogoliubov model. Phys. Rev. C 2003, 67, 034312. [CrossRef]

28. Klüpfel, P.; Reinhard, P.G.; Bürvenich, T.J.; Maruhn, J.A. Variations on a theme by Skyrme: A systematic study of adjustments of model parameters. Phys. Rev. C 2009, 79, 034310. [CrossRef]

29. Nikšić, T.; Paar, N.; Vretenar, D.; Ring, P. DIRHB-A relativistic self-consistent mean-field framework for atomic nuclei. Comput. Phys. Commun. 2014, 185, 1808-1821. [CrossRef]

30. Paar, N.; Vretenar, D.; Ring, P. Proton Electric Pygmy Dipole Resonance. Phys. Rev. Lett. 2005, 94, 182501. [CrossRef] 
31. Paar, N.; Niu, Y.F.; Vretenar, D.; Meng, J. Isoscalar and Isovector Splitting of Pygmy Dipole Structures. Phys. Rev. Lett. 2009, 103, 032502. [CrossRef] [PubMed]

32. Niu, Y.F.; Niu, Z.M.; Paar, N.; Vretenar, D.; Wang, G.H.; Bai, J.S.; Meng, J. Pairing transitions in finite-temperature relativistic Hartree-Bogoliubov theory. Phys. Rev. C 2013, 88, 034308. [CrossRef]

33. Niu, Y.; Paar, N.; Vretenar, D.; Meng, J. Low-energy monopole and dipole response in nuclei at finite temperature. Phys. Lett. B 2009, 681, 315-319. [CrossRef]

34. Khan, E.; Paar, N.; Vretenar, D. Low-energy monopole strength in exotic nickel isotopes. Phys. Rev. C 2011, 84, 051301. [CrossRef]

35. Yüksel, E.; Paar, N.; Colò, G.; Khan, E.; Niu, Y.F. Gamow-Teller excitations at finite temperature: Competition between pairing and temperature effects. Phys. Rev. C 2020, 101, 044305. [CrossRef]

36. Kružić, G.; Oishi, T.; Vale, D.; Paar, N. Magnetic dipole excitations based on the relativistic nuclear energy density functional. Phys. Rev. C 2020, 102, 044315. [CrossRef]

37. Paar, N.; Vretenar, D.; Marketin, T.; Ring, P. Inclusive charged-current neutrino-nucleus reactions calculated with the relativistic quasiparticle random-phase approximation. Phys. Rev. C 2008, 77, 024608. [CrossRef]

38. Samana, A.R.; Krmpotić, F.; Paar, N.; Bertulani, C.A. Neutrino and antineutrino charge-exchange reactions on ${ }^{12}$ C. Phys. Rev. C 2011, 83, 024303. [CrossRef]

39. Fantina, A.F.; Khan, E.; Colò, G.; Paar, N.; Vretenar, D. Stellar electron-capture rates on nuclei based on a microscopic Skyrme functional. Phys. Rev. C 2012, 86, 035805. [CrossRef]

40. Vale, D.; Rauscher, T.; Paar, N. Hybrid method to resolve the neutrino mass hierarchy by supernova (anti)neutrino induced reactions. J. Cosmol. Astropart. Phys. 2016, 2016, 007. [CrossRef]

41. Petković, J.; Marketin, T.; Martínez-Pinedo, G.; Paar, N. Self-consistent calculation of the reactor antineutrino spectra including forbidden transitions. J. Phys. G Nucl. Part. Phys. 2019, 46, 085103. [CrossRef]

42. Nikšić, T.; Vretenar, D.; Finelli, P.; Ring, P. Relativistic Hartree-Bogoliubov model with density-dependent meson-nucleon couplings. Phys. Rev. C 2002, 66, 024306. [CrossRef]

43. Tian, Y.; Ma, Z.y.; Ring, P. Axially deformed relativistic Hartree Bogoliubov theory with a separable pairing force. Phys. Rev. C 2009, 80, 024313. [CrossRef]

44. Finelli, P.; Kaiser, N.; Vretenar, D.; Weise, W. Relativistic nuclear model with point-couplings constrained by QCD and chiral symmetry. Nucl. Phys. A 2004, 735, 449-481. [CrossRef]

45. Bender, M.; Rutz, K.; Reinhard, P.G.; Maruhn, J.A. Pairing gaps from nuclear mean-field models. Eur. Phys. J. A 2000, 8, 59-75. [CrossRef]

46. Huang, W.; Audi, G.; Wang, M.; Kondev, F.G.; Naimi, S.; Xu, X. The AME2016 atomic mass evaluation (I). Evaluation of input data; and adjustment procedures. Chin. Phys. C 2017, 41, 030002. [CrossRef]

47. Wang, M.; Audi, G.; Kondev, F.G.; Huang, W.; Naimi, S.; Xu, X. The AME2016 atomic mass evaluation (II). Tables, graphs and references. Chin. Phys. C 2017, 41, 030003. [CrossRef]

48. Angeli, I.; Marinova, K. Table of experimental nuclear ground state charge radii: An update. At. Data Nucl. Data Tables 2013, 99, 69-95. [CrossRef]

49. Roca-Maza, X.; Paar, N.; Colò, G. Covariance analysis for energy density functionals and instabilities. J. Phys. G Nucl. Part. Phys. 2015, 42, 034033. [CrossRef]

50. Klähn, T.; Blaschke, D.; Typel, S.; van Dalen, E.N.E.; Faessler, A.; Fuchs, C.; Gaitanos, T.; Grigorian, H.; Ho, A.; Kolomeitsev, E.E.; et al. Constraints on the high-density nuclear equation of state from the phenomenology of compact stars and heavy-ion collisions. Phys. Rev. C 2006, 74, 035802. [CrossRef]

51. Drischler, C.; Furnstahl, R.J.; Melendez, J.A.; Phillips, D.R. How Well Do We Know the Neutron-Matter Equation of State at the Densities Inside Neutron Stars? A Bayesian Approach with Correlated Uncertainties. Phys. Rev. Lett. 2020, 125, 202702. [CrossRef] [PubMed]

52. Paar, N.; Vretenar, D.; Khan, E.; Colò, G. Exotic modes of excitation in atomic nuclei far from stability. Rep. Prog. Phys. 2007, 70, 691-793. [CrossRef]

53. Trzcińska, A.; Jastrzȩbski, J.; Lubiński, P.; Hartmann, F.J.; Schmidt, R.; von Egidy, T.; Kłos, B. Neutron Density Distributions Deduced from Antiprotonic Atoms. Phys. Rev. Lett. 2001, 87, 082501. [CrossRef] [PubMed]

54. Świątecki, W.J.; Trzcińska, A.; Jastrzębski, J. Difference of the root-mean-square sizes of neutron and proton distributions in nuclei: Comparison of theory with data. Phys. Rev. C 2005, 71, 047301. [CrossRef]

55. Birkhan, J.; Miorelli, M.; Bacca, S.; Bassauer, S.; Bertulani, C.A.; Hagen, G.; Matsubara, H.; von Neumann-Cosel, P.; Papenbrock, T.; Pietralla, N.; et al. Electric Dipole Polarizability of ${ }^{48}$ Ca and Implications for the Neutron Skin. Phys. Rev. Lett. $2017,118,252501$. [CrossRef]

56. Rossi, D.M.; Adrich, P.; Aksouh, F.; Alvarez-Pol, H.; Aumann, T.; Benlliure, J.; Böhmer, M.; Boretzky, K.; Casarejos, E.; Chartier, M.; et al. Measurement of the Dipole Polarizability of the Unstable Neutron-Rich Nucleus ${ }^{68}$ Ni. Phys. Rev. Lett. 2013, 111, 242503. [CrossRef]

57. Hashimoto, T.; Krumbholz, A.M.; Reinhard, P.G.; Tamii, A.; von Neumann-Cosel, P.; Adachi, T.; Aoi, N.; Bertulani, C.A.; Fujita, H.; Fujita, Y.; et al. Dipole polarizability of ${ }^{120} \mathrm{Sn}$ and nuclear energy density functionals. Phys. Rev. C 2015, 92, 031305. [CrossRef]

58. Bassauer, S.; von Neumann-Cosel, P.; Reinhard, P.G.; Tamii, A.; Adachi, S.; Bertulani, C.; Chan, P.; Colò, G.; DÁlessio, A.; Fujioka, H.; et al. Evolution of the dipole polarizability in the stable tin isotope chain. Phys. Lett. B 2020, 810, 135804. [CrossRef] 
59. Tamii, A.; Poltoratska, I.; von Neumann-Cosel, P.; Fujita, Y.; Adachi, T.; Bertulani, C.A.; Carter, J.; Dozono, M.; Fujita, H.; Fujita, K.; et al. Complete Electric Dipole Response and the Neutron Skin in ${ }^{208} \mathrm{~Pb}$. Phys. Rev. Lett. 2011, 107, 062502. [CrossRef]

60. Roca-Maza, X.; Viñas, X.; Centelles, M.; Agrawal, B.K.; Colò, G.; Paar, N.; Piekarewicz, J.; Vretenar, D. Neutron skin thickness from the measured electric dipole polarizability in ${ }^{68} \mathrm{Ni},{ }^{120} \mathrm{Sn}$, and ${ }^{208} \mathrm{~Pb}$. Phys. Rev. C 2015, 92, 064304. [CrossRef]

61. De Vries, H.; De Jager, C.; De Vries, C. Nuclear charge-density-distribution parameters from elastic electron scattering. At. Data Nucl. Data Tables 1987, 36, 495-536. [CrossRef]

62. Fricke, G.; Bernhardt, C.; Heilig, K.; Schaller, L.; Schellenberg, L.; Shera, E.; Dejager, C. Nuclear Ground State Charge Radii from Electromagnetic Interactions. At. Data Nucl. Data Tables 1995, 60, 177-285. [CrossRef]

63. Zumbro, J.D.; Shera, E.B.; Tanaka, Y.; Bemis, C.E.; Naumann, R.A.; Hoehn, M.V.; Reuter, W.; Steffen, R.M. E2 and E4 Deformations in $233,234,235,238$ U. Phys. Rev. Lett. 1984, 53, 1888-1892. [CrossRef]

64. Zumbro, J.; Naumann, R.; Hoehn, M.; Reuter, W.; Shera, E.; Bemis, C.; Tanaka, Y. E2 and E4 deformations in 232Th and 239,240,242Pu. Phys. Lett. B 1986, 167, 383-387. [CrossRef]

65. Ray, L. Neutron isotopic density differences deduced from $0.8 \mathrm{GeV}$ polarized proton elastic scattering. Phys. Rev. C 1979, 19, 1855-1872. [CrossRef]

66. Krasznahorkay, A.; Akimune, H.; van den Berg, A.; Blasi, N.; Brandenburg, S.; Csatloś, M.; Fujiwara, M.; Gulyaś, J.; Harakeh, M.; Hunyadi, M.; et al. Neutron-skin thickness in neutron-rich isotopes. Nucl. Phys. A 2004, 731, 224-234. [CrossRef]

67. Krasznahorkay, A.; Fujiwara, M.; van Aarle, P.; Akimune, H.; Daito, I.; Fujimura, H.; Fujita, Y.; Harakeh, M.N.; Inomata, T.; Jänecke, J.; et al. Excitation of Isovector Spin-Dipole Resonances and Neutron Skin of Nuclei. Phys. Rev. Lett. 1999, 82, 3216-3219. [CrossRef]

68. Klimkiewicz, A.; Paar, N.; Adrich, P.; Fallot, M.; Boretzky, K.; Aumann, T.; Cortina-Gil, D.; Pramanik, U.D.; Elze, T.W.; Emling, H.; et al. Nuclear symmetry energy and neutron skins derived from pygmy dipole resonances. Phys. Rev. C 2007, 76, 051603. [CrossRef]

69. Terashima, S.; Sakaguchi, H.; Takeda, H.; Ishikawa, T.; Itoh, M.; Kawabata, T.; Murakami, T.; Uchida, M.; Yasuda, Y.; Yosoi, M.; et al. Proton elastic scattering from tin isotopes at $295 \mathrm{MeV}$ and systematic change of neutron density distributions. Phys. Rev. C 2008, 77, 024317. [CrossRef]

70. Oishi, T.; Kružić, G.; Paar, N. Role of residual interaction in the relativistic description of M1 excitation. J. Phys. G Nucl. Part. Phys. 2020, 47, 115106. [CrossRef]

71. Ring, P.; Schuck, P. The Nuclear Many-Body Problems; Springer: Berlin/Heidelberg, Germany, 1980.

72. Fujita, Y.; Rubio, B.; Gelletly, W. Spin-isospin excitations probed by strong, weak and electro-magnetic interactions. Prog. Part. Nucl. Phys. 2011, 66, 549-606. [CrossRef]

73. Von Neumann-Cosel, P.; Poves, A.; Retamosa, J.; Richter, A. Magnetic dipole response in nuclei at the $\mathrm{N}=28$ shell closure: A new look1Work supported by the DFG under Contract Ri 242/12-1 and by research grants from the DGES (Spain), Nos. PB96-53 and PB96-604.1. Phys. Lett. B 1998, 443, 1-6. [CrossRef]

74. Vesely, P.; Kvasil, J.; Nesterenko, V.O.; Kleinig, W.; Reinhard, P.G.; Ponomarev, V.Y. Skyrme random-phase-approximation description of spin-flip M1 giant resonance. Phys. Rev. C 2009, 80, 031302. [CrossRef]

75. Nesterenko, V.O.; Kvasil, J.; Vesely, P.; Kleinig, W.; Reinhard, P.G.; Ponomarev, V.Y. Spin-flip M1 giant resonance as a challenge for Skyrme forces. J. Phys. G Nucl. Part. Phys. 2010, 37, 064034. [CrossRef]

76. Oishi, T.; Paar, N. Magnetic dipole excitation and its sum rule in nuclei with two valence nucleons. Phys. Rev. C 2019, 100, 024308. [CrossRef]

77. Birkhan, J.; Matsubara, H.; von Neumann-Cosel, P.; Pietralla, N.; Ponomarev, V.Y.; Richter, A.; Tamii, A.; Wambach, J. Electromagnetic M1 transition strengths from inelastic proton scattering: The cases of ${ }^{48} \mathrm{Ca}$ and ${ }^{208} \mathrm{~Pb}$. Phys. Rev. C 2016, $93,041302$. [CrossRef]

78. Tompkins, J.R.; Arnold, C.W.; Karwowski, H.J.; Rich, G.C.; Sobotka, L.G.; Howell, C.R. Measurements of the ${ }^{48}$ Ca $(\gamma, n)$ reaction. Phys. Rev. C 2011, 84, 044331. [CrossRef]

79. Mathy, M.; Birkhan, J.; Matsubara, H.; von Neumann-Cosel, P.; Pietralla, N.; Ponomarev, V.Y.; Richter, A.; Tamii, A. Search for weak M1 transitions in ${ }^{48}$ Ca with inelastic proton scattering. Phys. Rev. C 2017, 95, 054316. [CrossRef]

80. Laszewski, R.M.; Rullhusen, P.; Hoblit, S.D.; LeBrun, S.F. Giant M1 Resonance in Pb. Phys. Rev. Lett. 1985, 54, 530-533. [CrossRef]

81. Laszewski, R.M.; Alarcon, R.; Dale, D.S.; Hoblit, S.D. Distribution of M1 Transitions in ${ }^{208}$ Pb. Phys. Rev. Lett. 1988, 61, 1710-1712. [CrossRef] 